\title{
Spinning Loop Black Holes
}

\author{
Francesco Caravelli \\ Perimeter Institute for Theoretical Physics, 31 Caroline St., Waterloo, ON, N2L 2Y5, Canada and \\ Department of Physics and Astronomy, University of Waterloo, Waterloo, ON, N2L 3G1, Canada \\ Leonardo Modesto \\ Perimeter Institute for Theoretical Physics, 31 Caroline St., Waterloo, ON, N2L 2Y5, Canada
}

(Dated: October 30, 2018)

\begin{abstract}
In this paper we construct four Kerr-like spacetimes starting from the loop black hole Schwarzschild solutions (LBH) and applying the Newman-Janis transformation. In previous papers the Schwarzschild LBH was obtained replacing the Ashtekar connection with holonomies on a particular graph in a minisuperspace approximation which describes the black hole interior. Starting from this solution, we use a Newman-Janis transformation and we specialize to two different and natural complexifications inspired from the complexifications of the Schwarzschild and ReissnerNordström metrics. We show explicitly that the space-times obtained in this way are singularity free and thus there are no naked singularities. We show that the transformation move, if any, the causality violating regions of the Kerr metric far from $r=0$. We study the space-time structure with particular attention to the horizons shape. We conclude the paper with a discussion on a regular Reissner-Nordström black hole derived from the Schwarzschild LBH and then applying again the Newmann-Janis transformation.
\end{abstract}

PACS numbers: 04.60.Pp, 04.70.-s.

Keywords: Black hole, loop quantum gravity.

Contents

\section{Introduction}

II. The regular Schwarzschild-metric

III. The Newman-Janis algorithm

IV. Semi-polymeric spinning LBH

A. Type I complexification

B. Type II complexification

C. Closed time-like curves

V. The full-polymeric spinning LBH

A. Violation of causality

B. Horizon transitions

VI. Towards spinning LBH with charge

A. Reissner-Nordström LBH

1. Semi-polymeric case

2. Full-polymeric case

B. Kerr-Newmann LBH

1. Semi-polymeric case

2. Full-polymeric case

VII. Conclusions

\section{Acknowledgments}

\section{Appendices}

A. Kretschmann invariant for the Type I semi-polymeric LBH

B. Tortoise coordinates for the full polymeric metric
References
1

2

3

$\frac{4}{8}$

10

13

13

14

14

14

14

14

15

15

\section{INTRODUCTION}

The quantization of gravity is one of the still open issues in theoretical physics. Several researchers with several different approaches are trying to achieve this target. Loop quantum gravity [1] is the most conservative one from this point of view, being based on the Dirac quantization and not relying on any exotic idea. However in this context it is difficult to study the semiclassical regime of the theory and the classical limit. While several progresses have been made in the context of spin foams, still lot of work is needed in order to control the theory [2]. However, one of the successes of general relativity is its geometric content and it could be useful to keep a way to deal with the quantum using old and well known techniques.

In the context of polymeric quantization this point of view is truly the dominant one. In Loop Quantum Black Holes inspired by Loop Quantum Cosmology [3] , for instance, the quantum relies on a strong energy condition violating effective stress-energy tensor. The same techniques of Loop Quantum Cosmology have been used in the context of Polymeric Black Holes where we have a rich literature [4], [5], [6]. In this paper in particular we concentrate on the regular Schwarzschild metric that has been found in [4] within the minisuperspace approximation. This metric has several interesting properties, first of all the resolution of the singularity, the non expected self-duality property and the stability of the Cauchy horizon. It would be then interesting at this point to go a step forward and study black holes which possesses an 
asymptotic notion of angular momentum. Within GR such black hole has been found by Roy Kerr in the sixties and today it is well known as the Kerr spacetime; its eletrically charged counterpart is known as the KerrNewman solution. However these solutions are known to be not physical inside to horizon for the presence, as for instance in the Kerr case, of time machines close to the ring singularity. Being such, we can ask if such behavior persists when addressing the resolution of the Kerr ring singularity problem in the context of Loop Black Holes. Unfortunately it is known that finding such type of solution is not an easy task. In the context of loop black holes not even some sort of simplified equations are known. Thus we are facing the exceptional task of finding a solution for a spacetime without even having the differential equations to solve in order to find it. Being such the situation we can try to use a trick to find a solution that would be otherwise impossible to obtain. Such trick has been known for long time as the NewmanJanis transformation in general relativity. As we will see one of the steps of this transformation, the complexifica- tion step, is totally arbitrary. However, with a particular choice of the complexification, the algorithm gives both the Kerr and the Kerr-Newman solution starting from the Schwarzschild and the Reissner-Nordström respectively. It is then not surprising that when applied to the polymeric Schwarzschild black hole the solutions become the ordinary Kerr metric if we put the polimeric parameters and/or $l_{P}$ (the Planck length) to zero. In this paper we will use the Newman-Janis algorithm to construct four different rotating spacetimes.

In this paper we consider two spherically symmetric space-time that we call semi-polymeric and fullpolymeric. In [4] $\mathrm{LBH}$ solutions were obtained replacing the Ashtekar connection with holonomies on a particular graph in a minisuperspace approximation which describes the black hole interior. We call semi-polymeric the solution which is polymeric only in the radial component of the connection and full-polymeric the solution obtained replacing all the connection components with holonomies. The two LBH solutions are :

$$
\begin{aligned}
& \text { SEMI - POLYMERIC : } \quad d s^{2}=\frac{r^{3}(r-2 m)}{r^{4}+a_{o}^{2}} d t^{2}-\frac{d r^{2}}{\frac{r^{3}(r-2 m)}{r^{4}+a_{o}^{2}}}-\left(r^{2}+\frac{a_{o}^{2}}{r^{2}}\right) d \Omega^{(2)}, \\
& \text { FULL - POLYMERIC }: \quad d s^{2}=\frac{\left(r-r_{+}\right)\left(r-r_{-}\right)\left(r+r_{*}\right)^{2}}{r^{4}+a_{o}^{2}} d t^{2}-\frac{d r^{2}}{\frac{\left(r-r_{+}\right)\left(r-r_{-}\right) r^{4}}{\left(r+r_{*}\right)^{2}\left(r^{4}+a_{o}^{2}\right)}}-\left(r^{2}+\frac{a_{o}^{2}}{r^{2}}\right) d \Omega^{(2)},
\end{aligned}
$$

where we used signature: $(+,-,-,-)$. In section II and IV we will give a few more details about the solutions.

We will start from the semi-polymeric metric and choose the two most natural complexification of the metric compatible with the complexification of the Schwarzschild metric. We will discuss the same procedure in the full-polymeric case.

With the risk of being repetitive, the second aim of the paper is the following and is here stressed. The Kerr solution is considered as non physical inside the horizon due to the presence of closed time-like curves (CTC) when the metric is geodetically extended in the negative radial regions. For this reason Penrose long ago considered the possibility of a cosmic censorship avoiding the creation of naked singularities in general relativity. Being that the CTC's are close to the ring singularity of the Kerr metric, it is interesting to study geometries that are asymptotically Kerr-like but resolve the ring singularity problem. Recently Smailagic and Spallucci [7] found the equivalent of the Kerr black hole in the scenario of noncommutative inspired black holes introduced by Nicolini and Spallucci [8]. Such solution has no ring singularity, no superluminal motion and no CTC's and so can be considered as a physical solution. The ring singularity is replaced by a rotating classical string which puts in rotation the space- time. It is then interesting for us to study the space-times obtained from the LBH with the NJ algorithm and check if still there are causality violating regions.

The structure of the paper is the following: in section II we briefly recall the properties of the Schwarzschild loop black hole; in section III we review the Newman-Janis transformation; in section IV we apply the transformation to the semi-polymeric Schwarzschild loop black hole and we study the CTC's for these two metrics; in section $\mathrm{V}$ we apply the transformation to the full-polymeric Schwarzschild metric. In section VI we introduce the electric charge in the spherically symmetric LBHs and we apply again the Newmann-Janis transformation to construct the Kerr-Newmann space-time; conclusions follow. In the paper we use natural units $c=G=\hbar=1$.

\section{THE REGULAR SCHWARZSCHILD-METRIC}

Let us first summarize the regular black hole metric [4] that will be the starting point in the following. The solution is obtained from the canonical quantization of the Einstein equations written in terms of the Ashtekar variables, that is in terms of an $\mathrm{SU}(2)$ 3-dimensional con- 
nection $A$ and a triad $E$. The result is that the basis states of LQG are closed graphs the edges of which are labelled by irreducible $\mathrm{SU}(2)$ representations and the vertices by $\mathrm{SU}(2)$ intertwiners. Physically, the edges represent quanta of area with area $\gamma l_{\mathrm{P}}^{2} \sqrt{j(j+1)}$, where $j$ is the representation label on the edge (a half-integer), $l_{\mathrm{P}}$ is the Planck length, and $\gamma$ is a parameter of order 1 called the Immerzi parameter. Vertices of the graph represent quanta of 3-volume. The important observation to make here is that area is quantized and the smallest quanta of area possible has area $\sqrt{3} / 2 \gamma l_{\mathrm{P}}^{2}$.

The regular black hole metric that we will be using is derived from a simplified model of LQG [4]. To obtain this simplified model we make the following assumptions. First of all, the number of variables is reduced by assuming spherical symmetry. Then, instead of all possible closed graphs, a regular lattice with edge lengths $\delta_{b}$ and $\delta_{c}$ is used. The solution is then obtained dynamically inside the homogeneous region (inside the horizon where space is homogeneous but not static). Analytically continuing the solution outside the horizon one finds that one can reduce the two free parameters by imposing that the minimum area presents in the solution corresponds to the minimum area of LQG. The one remaining unknown constant $\delta_{b}$ is a parameter of the model determining the strength of deviations from the classical theory, and would have to be constrained by experiment. With the plausible expectation that the quantum graviational corrections become relevant only when the curvature is in the Planckian regime, corresponding to $\delta_{b}<1$, outside the horizon the solution is the Schwarzschild solution up to negligible Planck-scale corrections which allows us to believe the legitimacy of the analytical extension outside the horizon. The analytical extension is supported by a rigorous analysis explained in detail in [4].

This quantum gravitationally corrected Schwarzschild metric can be expressed in the form

$$
d s^{2}=G(r) d t^{2}-\frac{d r^{2}}{F(r)}-H(r) d \Omega^{2},
$$

with $d \Omega=d \theta^{2}+\sin ^{2} \theta d \phi^{2}$ and

$$
\begin{aligned}
& G(r)=\frac{\left(r-r_{+}\right)\left(r-r_{-}\right)\left(r+r_{*}\right)^{2}}{r^{4}+a_{o}^{2}}, \\
& F(r)=\frac{\left(r-r_{+}\right)\left(r-r_{-}\right) r^{4}}{\left(r+r_{*}\right)^{2}\left(r^{4}+a_{o}^{2}\right)}, \\
& H(r)=r^{2}+\frac{a_{o}^{2}}{r^{2}} .
\end{aligned}
$$

Here, $r_{+}=2 m$ and $r_{-}=2 m P^{2}$ are the two horizons, and $r_{*}=\sqrt{r_{+} r_{-}}=2 \mathrm{mP} . P$ is the polimeric function $P=$ $\left(\sqrt{1+\epsilon^{2}}-1\right) /\left(\sqrt{1+\epsilon^{2}}+1\right)$, with $\epsilon \ll 1$ the product of the Immirzi parameter $(\gamma)$ and the polimeric parameter $\left(\delta_{b}\right)$. With this, it is also $P \ll 1$, such that $r_{-}$and $r_{*}$ are very close to $r=0$. The area $a_{o}$ is equal to $A_{\min } / 8 \pi$, $A_{\text {min }}$ being the minimum area gap of LQG.

Note that in the above metric, $r$ is only asymptotically the usual radial coordinate since $g_{\theta \theta}$ is not just $r^{2}$.
This choice of coordinates however has the advantage of easily revealing the properties of this metric as we will see. But first, most importantly, in the limit $r \rightarrow \infty$ the deviations from the Schwarzschild-solution are of order $M \epsilon^{2} / r$, where $M$ is the usual ADM-mass:

$$
\begin{aligned}
& G(r) \rightarrow 1-\frac{2 M}{r}\left(1-\epsilon^{2}\right) \\
& F(r) \rightarrow 1-\frac{2 M}{r} \\
& H(r) \rightarrow r^{2}
\end{aligned}
$$

The ADM mass is the mass inferred by an observer at flat asymptotic infinity; it is determined solely by the metric at asymptotic infinity. The parameter $m$ in the solution is related to the mass $M$ by $M=m(1+P)^{2}$.

If one now makes the coordinate transformation $R=$ $a_{o} / r$ with the rescaling $\tilde{t}=t r_{*}^{2} / a_{o}$, and simultaneously substitutes $R_{ \pm}=a_{o} / r_{\mp}, R_{*}=a_{o} / r_{*}$ one finds that the metric in the new coordinates has the same form as in the old coordinates and thus exhibits a very compelling type of self-duality with dual radius $r=\sqrt{a_{o}}$. Looking at the angular part of the metric, one sees that this dual radius corresponds to a minimal possible surface element. It is then also clear that in the limit $r \rightarrow 0$, corresponding to $R \rightarrow \infty$, the solution does not have a singularity, but instead has another asymptotically flat Schwarzschild region.

The metric in Eq. (3) is a solution of a quantum gravitationally corrected set of equations which, in the absence of quantum corrections $\epsilon, a_{o} \rightarrow 0$, reproduces Einstein's field equations.

\section{THE NEWMAN-JANIS ALGORITHM}

In this section we review the Newman-Janis transformation for a generic spherically symmetric spacetime [9]. Roughly speaking, the algorithm start from a nonrotating spacetime and at the end of the steps the spacetime is rotating. We stress that one of the steps is arbitrary but can be constrained by the classical limit. The starting point a spherically symmetric spacetime. In its most general form, the metrics are of the following form,

$$
\begin{aligned}
d s^{2} & =e^{2 \Phi(r)} d t^{2}-e^{2 \lambda(r)} d r^{2}-H(r) d \Omega^{2} \\
& :=G(r) d t^{2}-\frac{d r^{2}}{F(r)}-H(r) d \Omega^{2}
\end{aligned}
$$

which define the function $\Phi(r)$ and $\lambda(r)$ used in literature with $G(r)$ and $F(r)$. The first step of the transformation is to change coordinates. This step requires the advanced null coordinates $\{u, r, \vartheta, \phi\}$, where

$$
u=t-r^{*}
$$

and $d r^{*}=d r / \sqrt{G F}$. The line element above then becomes,

$$
d s^{2}=G(r) d u^{2}+2 \sqrt{\frac{G(r)}{F(r)}} d u d r-H(r) d \Omega^{2},
$$


while the non zero components of the inverse metric are

$$
\begin{aligned}
& g^{u \phi}=e^{-\Phi(r)-\lambda(r)}, g^{\phi \phi}=-\left[H(r) \sin ^{2} \theta\right]^{-1}, \\
& g^{\theta \theta}=-H(r)^{-1}, g^{r r}=-e^{-2 \lambda(r)} .
\end{aligned}
$$

The second step of the algorithm is to find the null tetrads for the inverse matrix as follows,

$$
\begin{aligned}
& g^{\mu \nu}=l^{\mu} n^{\nu}+l^{\nu} n^{\mu}-m^{\mu} \bar{m}^{\nu}-m^{\nu} \bar{m}^{\mu} \\
& l^{\mu}=\delta_{r}^{\mu} \\
& n^{\mu}=\sqrt{\frac{F}{G}} \delta_{u}^{\mu}-\frac{1}{2} F \delta_{r}^{\mu}, \\
& m^{\mu}=\frac{1}{\sqrt{2 H}}\left(\delta_{\theta}^{\mu}+\frac{i}{\sin \theta} \delta_{\phi}^{\mu}\right) .
\end{aligned}
$$

where the vectors satisfy the relations $l_{\mu} l^{\mu}=m_{\mu} m^{\mu}=$ $n_{\mu} n^{\mu}=l_{\mu} m^{\mu}=n_{\mu} m^{\mu}=0$ and $l_{\mu} n^{\mu}=-m_{\mu} \bar{m}^{\mu}=1$ $(\bar{x}$ is the complex conjugate of the general quantity $x$ ). The main step of the procedure is the combination of two operations. The first complex transformation in the $r-u$ plane as follows:

$$
\begin{aligned}
& r \rightarrow r^{\prime}=r+i a \cos \theta, \\
& u \rightarrow u^{\prime}=u-i a \cos \theta .
\end{aligned}
$$

together with a complexification of the functions $F, G$ and $H$ of the metric, under which the null tetrads become

$$
\begin{aligned}
& l^{\mu}=\delta_{1}^{\mu} \\
& n^{\mu}=\sqrt{\frac{\tilde{F}\left(r^{\prime}\right)}{\tilde{G}\left(r^{\prime}\right)}} \delta_{u}^{\mu}-\frac{1}{2} \tilde{F}\left(r^{\prime}\right) \delta_{r}^{\mu} \\
& m^{\mu}=\frac{1}{\sqrt{2 \tilde{H}\left(r^{\prime}\right)}}\left(i a \sin \theta\left(\delta_{u}^{\mu}-\delta_{r}^{\mu}\right)+\delta_{\theta}^{\mu}+\frac{i}{\sin \theta} \delta_{\phi}^{\mu}\right) .
\end{aligned}
$$

where $\tilde{F}, \tilde{G}$ and $\tilde{H}$ are real functions on the complex domain. This step of the procedure is in principle completely arbitrary. In fact in the original paper Newman and Janis could not give a true explanation of the procedure if not that it works for the Kerr metric with a particular choice of the complexifications. The situation improved with Drake and Szekeres [9], in which they prove that the only Petrov D space-times generated by the Newman-Janis algorithm with a vanishing Ricci scalar is the Kerr-Newman spacetime. Having this fact in mind, the non zero components of the inverse metric (7) become, using the tetrads (11) after the transformation,

$$
\begin{aligned}
& g^{u u}=-\frac{a^{2} \sin ^{2}(\theta)}{\tilde{H}(r, \theta)}, g^{u \phi}=-\frac{a}{\tilde{H}(r, \theta)}, \\
& g^{\phi \phi}=-\frac{1}{\tilde{H}(r, \theta) \sin ^{2} \theta}, g^{\theta \theta}=-\frac{1}{\tilde{H}(r, \theta)}, \\
& g^{r r}=-\frac{a^{2} \sin ^{2} \theta}{\tilde{H}(r, \theta)}-e^{-2 \lambda(r, \theta)}, g^{r \phi}=\frac{a}{\tilde{H}(r, \theta)}, \\
& g^{u r}=\frac{a^{2} \sin ^{2}(\theta)}{\tilde{H}(r, \theta)}+e^{-\tilde{\Phi}(r, \theta)-\tilde{\lambda}(r, \theta)} .
\end{aligned}
$$

Let us now apply the procedure to the classical Schwarzschild example In this case the metric has $G=F$ or $\lambda=-\Phi$ and $H=r^{2}$. The metric reads, in usual Eddington-Finkelstein coordinates:

$$
\begin{aligned}
& d s^{2}=G(r) d u^{2}+2 d u d r-r^{2} d \Omega^{2} \\
& =\left(1-\frac{2 m}{r}\right) d u^{2}+2 d u d r-r^{2} d \Omega^{2}
\end{aligned}
$$

where $G(r)=1-\frac{2 m}{r}$ and we see that $H(r)=r^{2}$. If we apply the Newman-Janis algorithm as prescribed above, we have to choose a complexification of the $r^{2}$ and of the $1 / r$ term. In general this prescription is not unique. However since we know what the Kerr solution is, we know that if we take the following complexification:

$$
\begin{aligned}
& r^{2} \rightarrow r^{\prime} \bar{r}^{\prime}, \\
& \frac{1}{r} \rightarrow \frac{1}{2}\left(\frac{1}{r^{\prime}}+\frac{1}{\bar{r}^{\prime}}\right) .
\end{aligned}
$$

This trick works well. This is the same as complexifying in the following way the functions $G(r)$ and $H(r)$

$$
\begin{aligned}
& \tilde{G}\left(r^{\prime}\right)=1-m\left(\frac{1}{r^{\prime}}+\frac{1}{\bar{r}^{\prime}}\right)=1-\frac{2 m r}{r^{2}+a^{2} \cos ^{2} \theta}, \\
& \tilde{H}\left(r^{\prime}\right)=r^{\prime} \bar{r}^{\prime}=r^{2}+a^{2} \cos ^{2} \theta .
\end{aligned}
$$

in the classical Schwarzschild metric. The find metric is the Kerr metric in Kerr-Schild coordinates. However let us stress that in practice nothing could have told us, without using the Einstein equations, that a particular complexification is favored respect to the other one. This situation is even worse for Reissner-Nordström. In fact in this last case the function $G(r)$ is of the form:

$$
G(r)=1-\frac{2 m}{r}-\frac{Q^{2}}{r^{2}},
$$

where $\mathrm{Q}$ is the electric charge of the black hole. In this case the two most natural complexification of the last term in (14) is:

$$
\frac{1}{r^{2}} \rightarrow \frac{1}{r^{\prime} \bar{r}^{\prime}}
$$

After this introduction we can apply the procedure to the LBHs.

\section{SEMI-POLYMERIC SPINNING LBH}

The line element for the semi-polymeric black hole can be obtained from (3) setting $r_{*}=r_{-}=0$ and depends only on the mass and the minimum area $a_{o} \propto l_{P}^{2}\left(l_{P}=\right.$ $\sqrt{G_{N} \hbar}$ is the Planck length) (but can be obtained also from a simpler Hamiltonian constraint [4])

$$
\begin{aligned}
& d s^{2}=\frac{r^{3}(r-2 m)}{r^{4}+a_{o}^{2}} d t^{2}-\frac{d r^{2}}{\frac{r^{3}(r-2 m)}{r^{4}+a_{o}^{2}}}-H(r) d \Omega^{(2)} \\
& H(r)=r^{2}+\frac{a_{o}^{2}}{r^{2}} .
\end{aligned}
$$


This metric is regular everywhere and reproduces the Schwarzschild metric in the limit $a_{o} \rightarrow 0$. We can work with a more general form of the metric leaving the physical radius of the two sphere implicit

$$
d s^{2}=\frac{r^{2}\left(1-\frac{2 m}{r}\right)}{H(r)} d t^{2}-\frac{d r^{2}}{\frac{r^{2}\left(1-\frac{2 m}{r}\right)}{H(r)}}-H(r) d \Omega^{(2)} .
$$

and in Eddington-Finkelstein coordinates because $G=F$ (or $\Phi=-\lambda$ ) reduces to

$$
\begin{aligned}
d s^{2} & =\frac{r^{2}\left(1-\frac{2 m}{r}\right)}{H(r)} d u^{2}+2 d u d r-H(r) d \Omega^{2} \\
& =\frac{r^{2}\left(1-\frac{2 m}{r}\right)}{r^{4}+a_{o}^{2}} d u^{2}+2 d u d r-H(r) d \Omega^{2} .
\end{aligned}
$$

Now we complexify the functions $G$ and $H$ appearing in the metric. It is easy to understand that, in $G$, the term of the form $(1-2 m / r)$ must be complexified as (12) for compatibility with the Schwarzschild metric in the limit $a_{o}, a \rightarrow 0$. On the same footage the $r^{2}$ term in $H(r)$, for compatibility with Kerr in the $a_{o} \rightarrow 0$ limit. This means that in $G$ the $r^{2}$ must be complexified as $r^{2} \rightarrow r^{\prime} \bar{r}^{\prime}$ for compatibility with the Kerr metric in the limit $a_{o} \rightarrow 0$. Thus we are left only with the complexification of the $a_{o}$ term in $H$, which represent the quantum correction of the metric. The two most natural complexification of the term proportional to $a_{o}$ in $H(r)$ are:

$$
\text { Type I : } \quad \frac{a_{o}^{2}}{r^{2}} \rightarrow \frac{a_{o}^{2}}{\left(r^{\prime}+\bar{r}^{\prime}\right)^{2} / 4}
$$

or, as the Reissner-Nordström case suggests:

$$
\text { Type II : } \quad \frac{a_{o}^{2}}{r^{2}} \rightarrow \frac{a_{o}^{2}}{r^{\prime} \bar{r}^{\prime}}
$$

In the following we refer to complexification (19) as Type I and (20) as Type II.

\section{A. Type I complexification}

As explained earlier we proceed to complexify the components of the metric as

$$
\begin{aligned}
& G(r)=\frac{r^{2}\left(1-\frac{2 m}{r}\right)}{H(r)} \mapsto \tilde{G}\left(r^{\prime}\right):=G(r, \theta), \\
& \begin{aligned}
G(r, \theta) & =r^{\prime} \bar{r}^{\prime}\left[1-2 m \frac{1}{2}\left(\frac{1}{r^{\prime}}+\frac{1}{\bar{r}^{\prime}}\right)\right] \frac{1}{\tilde{H}\left(r^{\prime}\right)} \\
& =\left(\rho^{2}-2 m r\right) \frac{1}{\tilde{H}\left(r^{\prime}\right)}=\frac{\left(\rho^{2}-2 m r\right)}{\Sigma}, \\
H(r)= & r^{2}+\frac{a_{o}^{2}}{r^{2}} \mapsto \tilde{H}\left(r^{\prime}\right):=\Sigma(r, \theta), \\
\Sigma(r, \theta) & =r^{\prime} \bar{r}^{\prime}+\frac{a_{o}^{2}}{\left(r^{\prime}+\bar{r}^{\prime}\right)^{2} / 4}=\rho^{2}+\frac{a_{o}^{2}}{r^{2}}, \\
\rho^{2}(r, \theta) & :=r^{2}+a^{2} \cos ^{2} \theta .
\end{aligned}
\end{aligned}
$$
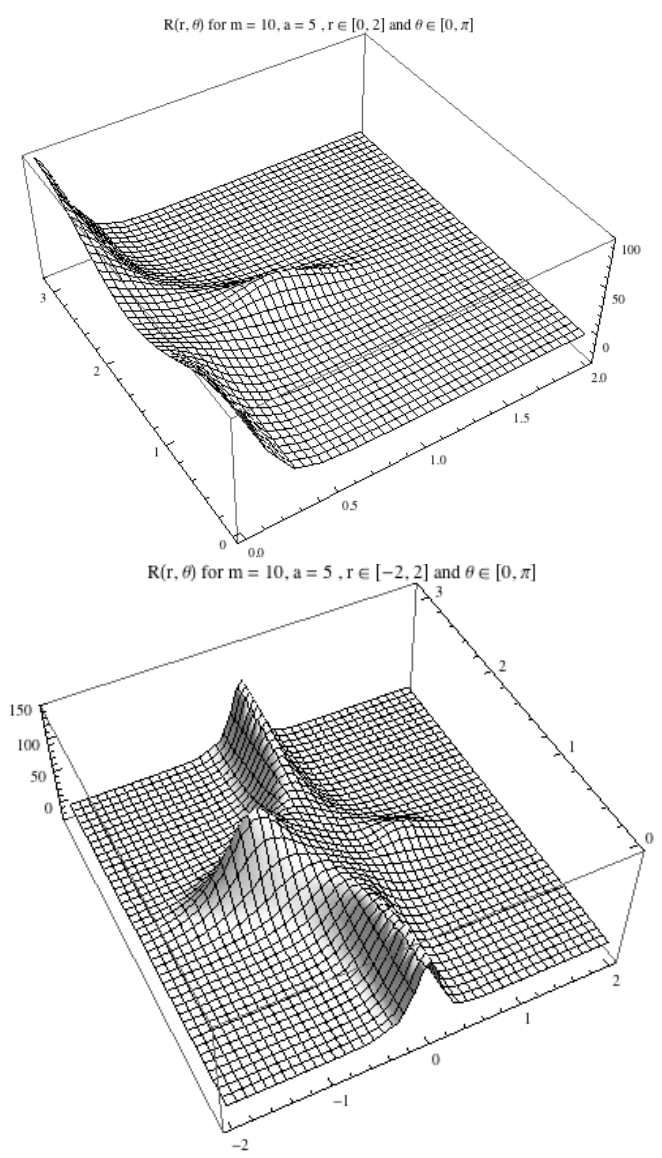

FIG. 1: Plot of the Ricci scalar for $m=10, a=5$ when the radial coordinate assume only positive values and for $r \in$ $[-2,2]$ in the second plot. Type I complexification.

in the original Kerr coordinates the metric reads

$$
\begin{aligned}
& d s_{(K)}^{2}=G(r, \theta) d u^{2}-\Sigma(r, \theta) d \theta^{2}-2 a \sin ^{2} \theta d r d \phi \\
& +\left[a^{2}(G(r, \theta)-2) \sin ^{2} \theta-\Sigma(r, \theta)\right] \sin ^{2}(\theta) d \phi^{2} \\
& +2 a(1-G(r, \theta)) \sin ^{2} \theta d \phi d u .
\end{aligned}
$$

In Kerr coordinates the metric is regular everywhere contrary to the classical one. There is no singularity on the event horizons and is simpler to show the regularity of this space-time. By a coordinate transformation the metric can be written in Boyer-Lindquist coordinates

$$
\begin{aligned}
& d s_{B-L}^{2}=G(r, \theta) d t^{2}-\frac{\Sigma(r, \theta) d r^{2}}{a^{2} \sin ^{2} \theta+G(r, \theta) \Sigma(r, \theta)} \\
& +2(1-G(r, \theta)) \sin ^{2} \theta d t d \phi-\Sigma(r, \theta) d \theta^{2} \\
& -\sin ^{2} \theta\left[a^{2}(2-G(r, \theta)) \sin ^{2} \theta+\Sigma(r, \theta)\right] d \phi^{2}
\end{aligned}
$$

In order to simplify the notation we introduce the following quantities

$$
\begin{aligned}
& \Delta(r)=G(r, \theta) \Sigma(r, \theta)+a^{2} \sin ^{2} \theta=r^{2}-2 m r+a^{2}, \\
& G(r, \theta)=\frac{\Delta(r)-a^{2} \sin ^{2} \theta}{\Sigma(r, \theta)},
\end{aligned}
$$


inside the metric and we write down the line element explicitly in Boyer-Lindquist (B-L) coordinates defined by the coordinate transformation $d u=d t+g(r) d r, d \phi=$ $d \phi^{\prime}+h(r) d r$ (we will omit the dependents on $\theta$ and $r$ in the function $\Delta, \Sigma)$ where

$$
\begin{aligned}
& g(r)=-\frac{e^{\lambda}\left(\Sigma+a^{2} e^{\lambda+\Phi}\right)}{e^{\Phi}\left(\Sigma+a^{2} \sin ^{2} \theta e^{2 \lambda}\right)}, \\
& h(r)=-\frac{a e^{2 \lambda}}{\Sigma+a^{2} \sin ^{2} \theta e^{2 \lambda}},
\end{aligned}
$$

are valid for general functions $\Phi$ and $\lambda$. The B-L metric reads

$$
\begin{aligned}
& d s^{2}=\frac{\Delta-a^{2} \sin ^{2} \theta}{\Sigma} d t^{2}-\frac{\Sigma}{\Delta} d r^{2} \\
& +2 a \sin ^{2} \theta\left(1-\frac{\Delta-a^{2} \sin ^{2} \theta}{\Sigma}\right) d t d \phi-\Sigma d \theta^{2} \\
& -\sin ^{2} \theta\left[\Sigma+a^{2} \sin ^{2} \theta\left(2-\frac{\Delta-a^{2} \sin ^{2} \theta}{\Sigma}\right)\right] d \phi^{2} .
\end{aligned}
$$

The first quantity we study is the Ricci scalar tensor which is classically zero in the empty space but not now, because of the quantum geometry effects,

$$
\begin{aligned}
& R(r, \theta)=\frac{8 a_{o}^{2}}{\left(a^{2} r^{2} \cos (2 \theta)+a^{2} r^{2}+2 a_{o}^{2}+2 r^{4}\right)^{3}} \times \\
& {\left[3 a^{4} r^{2}+a^{2} \cos (2 \theta)\left(3 r^{2}\left(a^{2}-2 m r\right)+a_{o}^{2}\right)\right.} \\
& \left.+3 a^{2}\left(a_{o}^{2}-2 m r^{3}+6 r^{4}\right)-4 r\left(a_{o}^{2}+3 r^{4}\right)(2 m-r)\right],
\end{aligned}
$$

which is zero for $a_{o} \rightarrow 0$. On the equatorial plane $(\theta=\pi / 2)$ and $r=0, R(0, \pi / 2)=2 a^{2} / a_{o}^{2}$. The plots in Fig 1 show the Ricci scalar is non singular and peaked in $\theta \approx \pi / 2, r \approx \sqrt{a_{o}}$. To complete the singularity resolution analysis we should analyze the regularity properties of the Kretschmann invariant tensor $K:=R_{\mu \nu \rho \sigma} R^{\mu \nu \rho \sigma}$. This quantity is given in the appendix but the regularity properties are shown with the tool of the two dimensional plots. The plots are given in Fig 213 where it is evident the metric is regular everywhere and the value of the curvature in $r=0, \theta=\pi / 2$ is $K(0, \pi / 2)=4 a^{4} / a_{o}^{4}$.

The component $g_{t t}$ of the metric changes sign on the surfaces defined by

$$
g_{t t}=0 \rightarrow \Delta(r)-a^{2} \sin ^{2} \theta=0,
$$

or more explicitly in

$$
r=m \pm \sqrt{m^{2}-a^{2} \cos ^{2} \theta},
$$

that define the ergosphere of the classical Kerr spacetime.

The horizons are those surfaces where the light cones lies and then also the light can not escape. In other words they are defined by

$$
\left(\frac{d r}{d t}\right)^{2}=0 \forall d \theta, d \phi .
$$

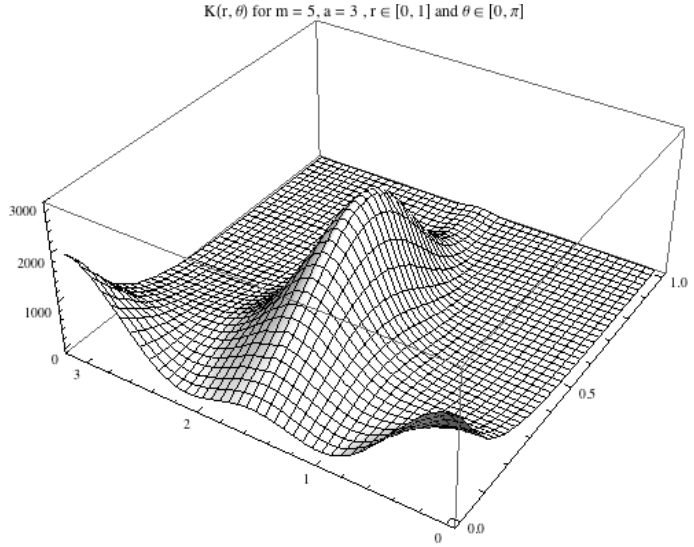

FIG. 2: Plot of the Kretschmann invariant for $(m, a)=(5,3)$ and $r \geqslant 0$ in Planck units. Type I complexification.

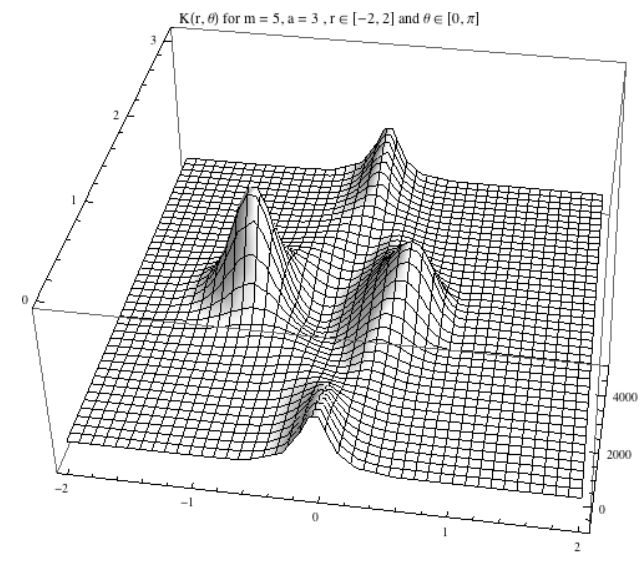

FIG. 3: Plot of the Kretschmann invariant for $(m, a)=(5,3)$ and positive and negative values of $r$ in Planck units. Type I complexification.

In our case this happens only where $\Delta(r)=0$ or

$$
r=r_{ \pm}:=m \pm \sqrt{m^{2}-a^{2}} \text { for } a<m .
$$

The event horizon is a null surface and a Killing surface as we are going to show. The surface $\Sigma(t, r, \theta, \phi)=$ const. is a null surface if the normal $n_{i}=\partial \mathcal{S} / \partial x^{i}$ is a null vector or satisfies the condition $n_{i} n^{i}=0$. The last identity says that the vector $n^{i}$ is on the surface $\mathcal{S}(t, r, \theta, \phi)$ itself, in fact $d \mathcal{S}=d x^{i} \partial \mathcal{S} / \partial x^{i}$ and $d x^{i} \| n^{i}$. The norm of the vector $n_{i}$ is

$$
n_{i} n^{i}=g^{i j} \frac{\partial \mathcal{S}}{\partial x^{i}} \frac{\partial \mathcal{S}}{\partial x^{i}}=0 .
$$

In our case (32) reduces to

$$
g^{r r} \frac{\partial \mathcal{S}}{\partial r} \frac{\partial \mathcal{S}}{\partial r}+g^{\theta \theta} \frac{\partial \mathcal{S}}{\partial \theta} \frac{\partial \mathcal{S}}{\partial \theta}=0 .
$$

and this equation is satisfied where $g^{r r}(r)=0$ if the surface is independent from $\theta, \mathcal{S}(r, \theta)=\mathcal{S}(r)$. The points 


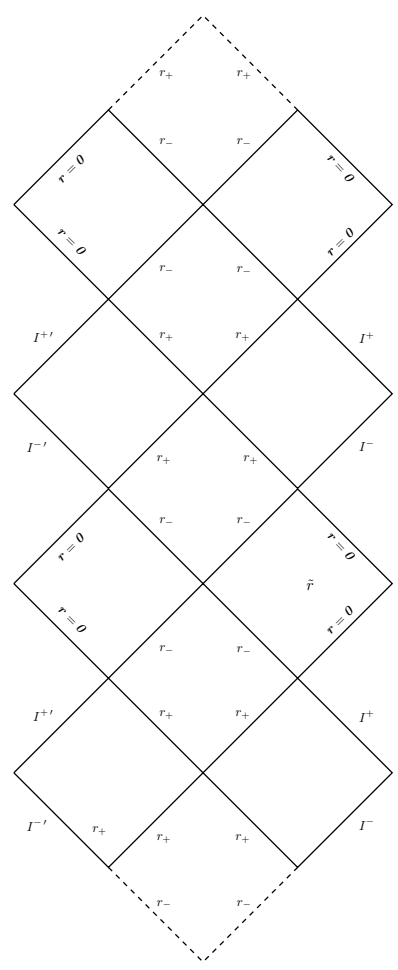

FIG. 4: Penrose diagram for $\theta=0$ and $r \geqslant 0$.

where $g^{r r}=0$ are $r_{+}$and $r_{-}$and $r=0$ but only $r_{-}$and $r_{+}$are horizons. The metric admits two killing vector $t^{\mu}=\partial_{t}$ and $\phi^{\mu}=\partial_{\phi}$ but also any linear combination is a Killing vector. In particular

$$
\xi^{\mu}=t^{\mu}+\Omega_{H} \phi^{\mu},
$$

is a Killing vector and for

$$
\Omega_{H}=\frac{a r_{+}^{2}}{r_{+}^{2}\left(a^{2}+r_{+}^{2}\right)+a_{o}^{2}}
$$

it is null on the event horizon $\left(r_{+}\right),\left.\xi^{\mu} \xi_{\mu}\right|_{r_{+}}=0$; this concludes the proof that the event horizon is a Killing horizon. We can calculate also the surface gravity on $r_{+}$ and $r_{-}$. It is defined in terms of the Killing vector (34) by

$$
\kappa^{2}=-\frac{1}{2} \nabla^{\mu} \xi^{\nu} \nabla_{\mu} \xi_{\nu}
$$

and the result is

$$
\begin{gathered}
\kappa_{+}=\frac{r_{+}^{2}\left(r_{+}-r_{-}\right)}{2\left(a^{2} r_{+}^{2}+a_{o}^{2}+r_{+}^{4}\right)}, \\
\kappa_{-}=\frac{r_{-}^{2}\left(r_{+}-r_{-}\right)}{4\left(a^{2} r_{-}^{2}+a_{o}^{2}+r_{-}^{4}\right)} .
\end{gathered}
$$

To conclude the event horizon area is
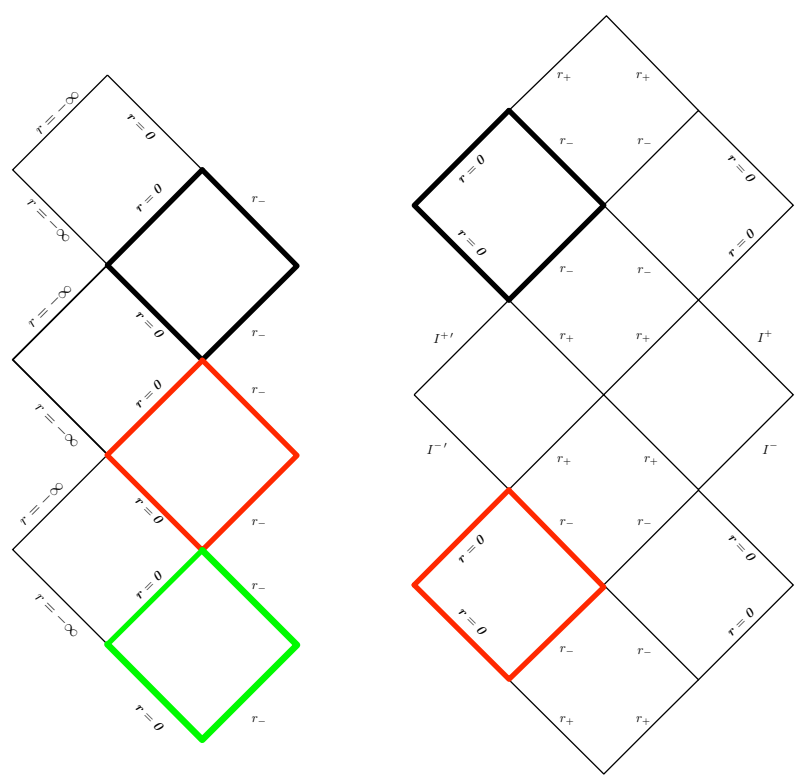

FIG. 5: Maximal extension of the Type I space-time for $a<m$ and $\theta=0$. The surface $r=0$ is a null surface but it is not an horizon beside $g_{t t}$ and $g_{r r}$ do not change sign. Block of the same color has to be identify to have the maximal extension of the space-time to negative value of $r$.

$$
A_{H}=4 \pi \frac{\left(r_{+}^{4}+a^{2} r_{+}^{2}+a_{o}^{2}\right)}{r_{+}^{2}} .
$$

The metric for $\theta$ and $\phi$ constant reduces to

$$
d s^{2}=\frac{\left(\Delta-a^{2} \sin ^{2} \theta_{o}\right)}{\Sigma} d t^{2}-\frac{\Sigma}{\Delta} d r^{2}
$$

and the tortoise coordinate for $\theta=0$ is

$$
\begin{aligned}
r^{*}=r & -\frac{a_{o}^{2}}{r r_{-} r_{+}}+\frac{a_{o}^{2}\left(r_{-}+r_{+}\right)}{r_{-}^{2} r_{+}^{2}} \log |r| \\
& +\frac{\left(a^{2} r_{-}^{2}+a_{o}^{2}+r_{-}^{4}\right)}{r_{-}^{2}\left(r_{-}-r_{+}\right)} \log \left|r-r_{-}\right| \\
& +\frac{\left(a^{2} r_{+}^{2}+a_{o}^{2}+r_{+}^{4}\right)}{r_{+}^{2}\left(r_{+}-r_{-}\right)} \log \left|r-r_{+}\right| .
\end{aligned}
$$

We can introduce first the coordinates $u=t-r^{*}$ and $v=t+r^{*}$ and then $U^{ \pm}=\mp \exp \left(\mp \kappa_{ \pm} u\right) / \kappa_{ \pm}, V^{ \pm}=$ $\pm \exp \left( \pm \kappa_{ \pm} v\right) / \kappa_{ \pm}$for $r>r_{-}$and $r<r_{-}$respectively. Looking to $U^{-} V^{-}=-\exp \left(-2 \kappa_{-} r^{*}\right) / \kappa_{-}^{2}$ we see that $U^{-} V^{-} \rightarrow 0$ for $r \rightarrow r_{-}$and $U^{-} V^{-} \rightarrow-\infty$ for $r=0$. The Penrose diagram in Fig 4 is a Penrose diagram for $r>0$. Despite the position of the point $r=0$ in the diagram it is not an event horizon as can be seen solving (30). A maximal extension to negative values of $r$ is obtained following the analysis in [10]. The result is given in Fig 5 .

As we showed studying the Ricci scalar and in particular the Kretschmann invariant the space-time is regular 


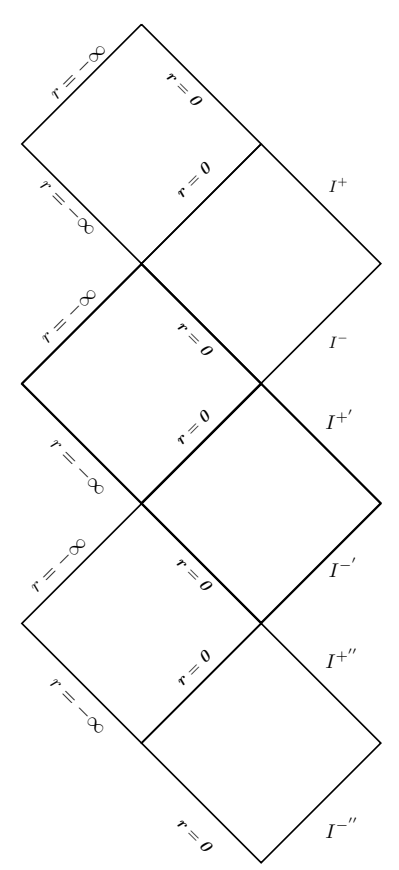

FIG. 6: Maximal extension of the Type I space-time for $a>m$ and $\theta=0$. The surface $r=0$ is a null surface but it is not an horizon beside $g_{t t}$ and $g_{r r}$ do not change sign.

everywhere. If we plot the Kretschmann invariant for the case $a>m$ we obtain plots similar to those in Fig 2314. In other words we do not have naked singularities. The tortoise coordinate for $a>m$ and $\theta=0$ is

$$
\begin{aligned}
& r^{*}=r-\frac{a_{o}^{2}}{a^{2} r}+\frac{2 a_{o}^{2} m \log |r|}{a^{4}} \\
& +\frac{\left(2 a^{4} m^{2}-a^{2} a_{o}^{2}+2 a_{o}^{2} m^{2}\right) \arctan \left(\frac{r-m}{\sqrt{a^{2}-m^{2}}}\right)}{a^{4} \sqrt{a^{2}-m^{2}}} \\
& +\frac{m\left(a^{4}-a_{o}^{2}\right)}{a^{4}} \log \left(a^{2}-2 m r+r^{2}\right)
\end{aligned}
$$

In this case, to understand the causal structure of the space-time, we can also introduce coordinates $(u, v)$ and then a single couple of new coordinates $(U, V)$ because there is just one coordinate singularity in $r=0$. The result is a block of space-time which extend from $+\infty$ to $r=0$. Following again [10] the maximal extension of the space-time is given in Fig 6 . For the extremal case $m=a$ and $\theta=0$ the tortoise coordinate is

$$
\begin{aligned}
r^{*}=r & -\frac{a_{o}^{2}}{r r_{o}^{2}}-\frac{a^{2} r_{o}^{2}+a_{o}^{2}+r_{o}^{4}}{r_{o}^{2}\left(r-r_{o}\right)}+\frac{2 a_{o}^{2}}{r_{o}^{3}} \log |r| \\
& +\frac{2\left(r_{o}^{4}-a_{o}^{2}\right)}{r_{o}^{3}} \log \left|r-r_{o}\right|
\end{aligned}
$$

and the Penrose diagram is in Fig:7.

Following the analysis of the classical Kerr metric [10] we have focused our attention to the axis of symmetry

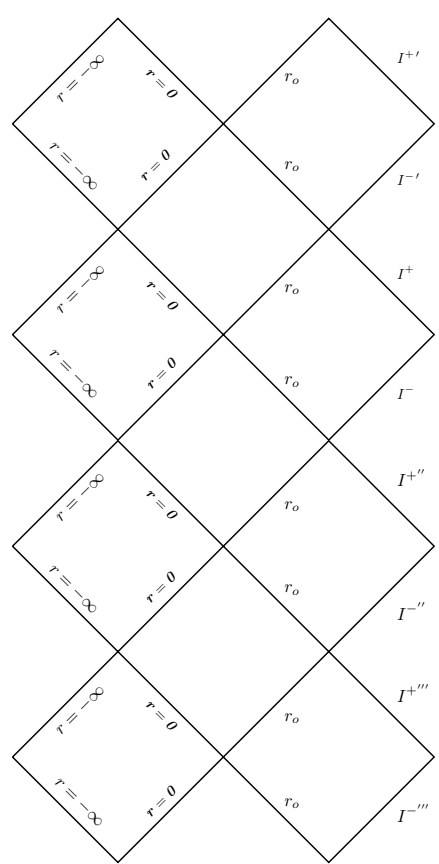

FIG. 7: Maximal extension of the Type I space-time for $a=m$ and $\theta=0\left(r_{o}=m\right)$.

because it is easier to study. Nevertheless it seems probable again as in the classical case that the basic topological properties of the four dimensional space-time are essentially the same.

\section{B. Type II complexification}

In this section we consider a different complexification starting from the same spherically symmetric metric (18) in Eddington-Finkelstein coordinates

$$
d s^{2}=\frac{r^{2}\left(1-\frac{2 m}{r}\right)}{H(r)} d u^{2}+2 d u d r-H(r) d \Omega^{2} .
$$

As explained the complexification (21) changes only in the factor $H(r)$,

$$
\begin{aligned}
& H(r) \mapsto \Sigma(r, \theta)=r^{\prime} \bar{r}^{\prime}+\frac{a_{o}^{2}}{r^{\prime} \bar{r}^{\prime}}=\rho^{2}+\frac{a_{o}^{2}}{\rho^{2}}, \\
& \rho^{2}(r, \theta):=r^{2}+a^{2} \cos ^{2} \theta .
\end{aligned}
$$

This small modification is sufficient to make the metric harder to study. In Kerr coordinates the metric is regular everywhere contrary to the classical one and is given in (22). There is no singularity on the event horizons and is more simple to show the regularity of the improved space-time metric. Again, the first quantity we study is the Ricci scalar tensor. The plots in Fig 89 show the Ricci scalar is non singular. To complete the singularity 


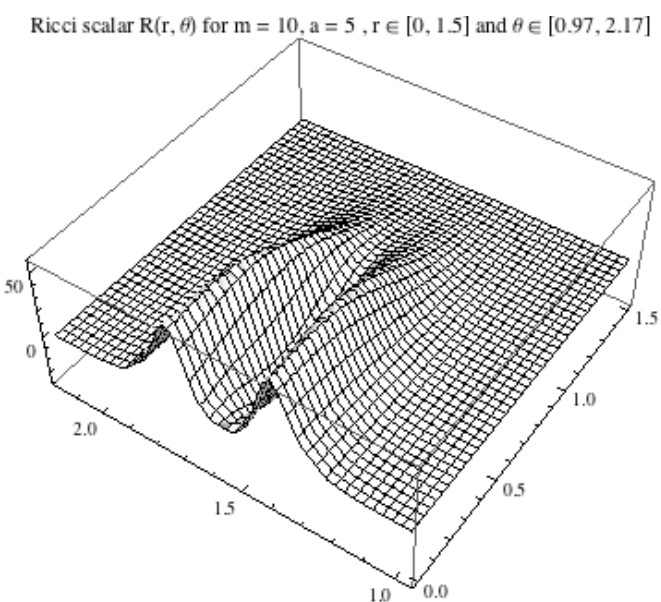

FIG. 8: Plot of the Ricci scalar for $m=10, a=5$ in Planck units $(r>0)$.

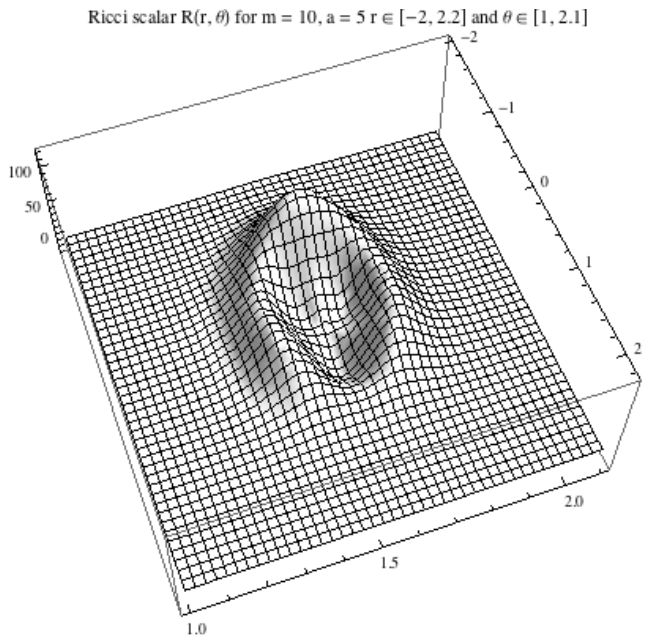

FIG. 9: Plot of the Ricci scalar for $m=10, a=5$ in Planck units and $r \in]-\infty,+\infty[$. The behavior at the origin is : $\lim _{\theta \rightarrow \pi / 2} \lim _{r \rightarrow 0} R(r, \theta)=\lim _{r \rightarrow 0} \lim _{\theta \rightarrow \pi / 2} R(r, \theta)=2 a^{2} / a_{o}^{2}$.

resolution analysis we should analyze the Kretschmann invariant tensor $K:=R_{\mu \nu \rho \sigma} R^{\mu \nu \rho \sigma}$. For this metric, more then for the type I, $K(r, \theta)$ is very involved and will show its regularity properties with the tool of three dimensional plots. The three dimensional plots are in Fig 101112 Passing to Boyer-Lindquist coordinates the metric assumes the same form of (26) but with a different function $\Sigma(r, \theta)$ defined in (21). Because $\Delta(r)$ is also the same the event horizon and the ergosphere surfaces are the same. The tortoise coordinate for $\theta=0$ and

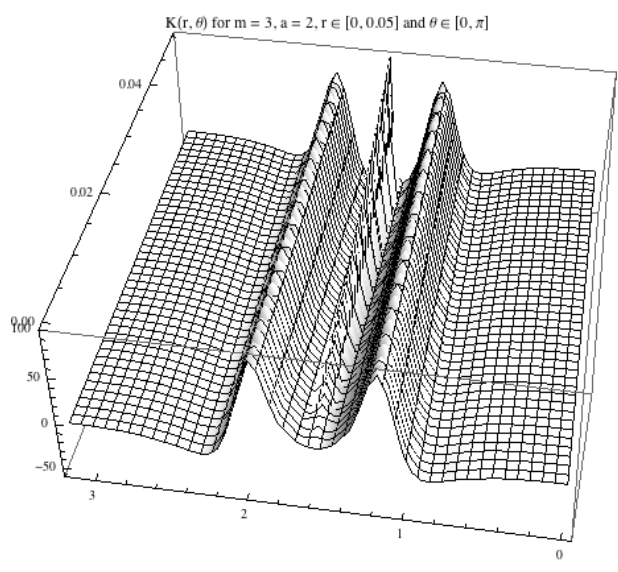

FIG. 10: Type II metric: Plot of the Kretschmann scalar for $m=3, a=2$ in Planck units near $r=0$.

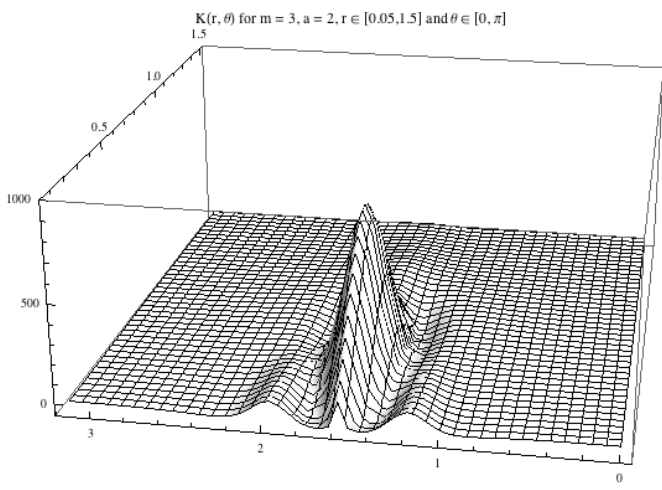

FIG. 11: Type II metric: Plot of the Kretschmann scalar for $m=3, a=2$ in Planck units for $r>0$.

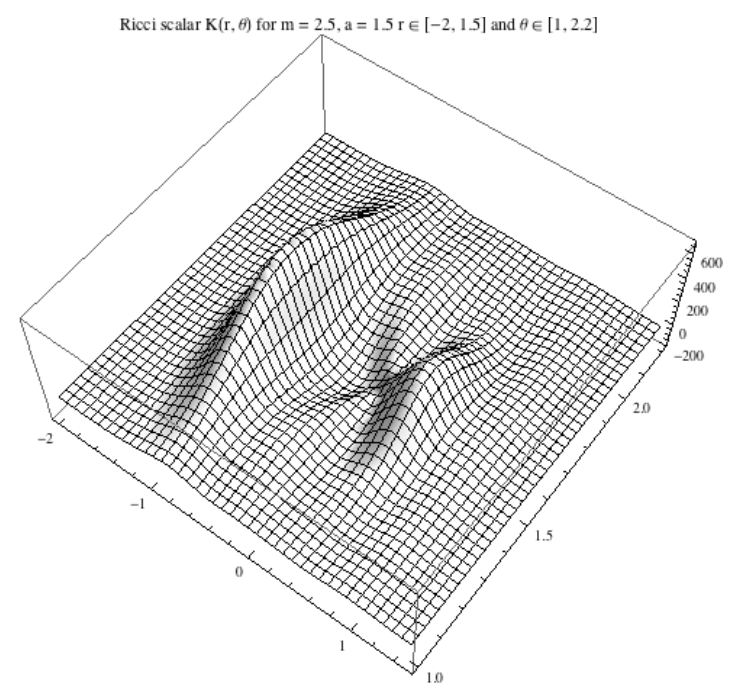

FIG. 12: Type II metric: Plot of the Kretschmann scalar for $m=3, a=2$ in Planck units. This plot involves also negative values of $r$ and the behavior at the origin is: $\lim _{\theta \rightarrow \pi / 2} \lim _{r \rightarrow 0} K(r, \theta)=\lim _{r \rightarrow 0} \lim _{\theta \rightarrow \pi / 2} K(r, \theta)=0$. 
$a<m$ is

$$
\begin{aligned}
r^{*}=r & +\frac{a_{o}^{2}\left(r_{-}+r_{+}\right)}{2\left(a^{2}+r_{-}^{2}\right)\left(a^{2}+r_{+}^{2}\right)} \log \left(a^{2}+r^{2}\right) \\
& -\frac{a_{o}^{2}\left(a^{2}-r_{-} r_{+}\right)}{a\left(a^{2}+r_{-}^{2}\right)\left(a^{2}+r_{+}^{2}\right)} \arctan (r / a) \\
& -\frac{\left(a^{4}+2 a^{2} r_{-}^{2}+a_{o}^{2}+r_{-}^{4}\right)}{\left(a^{2}+r_{-}^{2}\right)\left(r_{+}-r_{-}\right)} \log \left|r-r_{-}\right| \\
& +\frac{\left(a^{4}+2 a^{2} r_{+}^{2}+a_{o}^{2}+r_{+}^{4}\right)}{\left(a^{2}+r_{+}^{2}\right)\left(r_{+}-r_{-}\right)} \log \left|r-r_{+}\right| .
\end{aligned}
$$

Given the tortoise coordinate and using the same analysis of the previous section for the type I metric we can obtain the Penrose diagrams for the type II metric. In the $\theta=0$ case the diagrams are exactly the same of the classical Kerr metric for $a<m, a=m$ and $a>m$. There is no naked singularity and for $\theta=\pi / 2$ the diagram for the case $a>m$ looks like the Minkowski space-time diagram, as in the classical case, but with an extension to negative values of $r$.

\section{Closed time-like curves}

The metrics obtained in this section, as shown, have the nice properties of keeping the singularity free property of the original metric. Thus we can now see if the CTCs disappear in such space-times, since the ring singularity is not present anymore in both cases. In the Kerr case such CTCs are in the negatively $r$ geodetically extended space-time sector.

In order to study the CTC's problem we study the norm of the Killing vector along the $\phi$ direction. This vector has norm $\phi^{\mu} \phi_{\mu}=g_{\phi \phi}$. We calculate such norm for the classical metric and for the Type I and Type II metrics. We consider the norm near the point $r=0$, $\theta=\pi / 2$. Let $r / a=\delta$ (small and negative) and consider $\theta=\pi / 2+\delta$. Then classically we find

$$
\phi_{\mu} \phi^{\mu}=g_{\phi \phi}=-\frac{a m}{\delta}-a^{2}+\mathcal{O}(\delta),
$$

which is positive for sufficiently small and negative $\delta$. For the Type I and Type II loop black holes instead we find

$$
\begin{aligned}
\phi_{\mu} \phi^{\mu} & =-\frac{a_{o}^{2}}{a^{2} \delta^{2}}+\text { const. }+\mathcal{O}(\delta), \\
\phi_{\mu} \phi^{\mu} & =-\frac{a_{o}^{2}}{2 a^{2} \delta^{2}}+\text { const. }+\mathcal{O}(\delta)
\end{aligned}
$$

that are always negative for small values of $\delta$. We conclude there are no CTC in the region around $r \approx 0$ and $\theta \approx \pi / 2$ contrary to the classical Kerr space-time.

However for negative values of $r$ and arbitrary values of $\theta$ the norm of the Killing vector can change sign as showed in Fig 13 and we can still have CTC curves. The lump region in the plot is a time machine region [11].

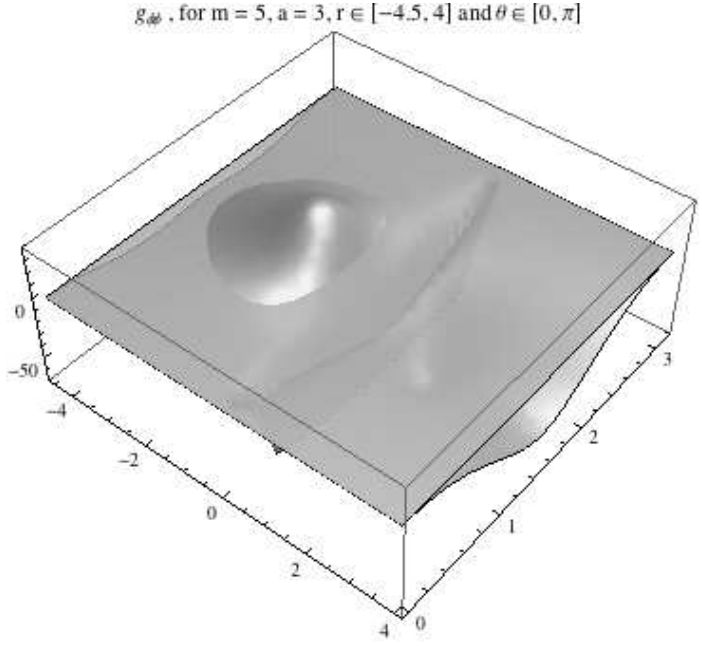

FIG. 13: In this plot the region where $g_{\phi \phi}$ is positive contains CTC curves in the analytical extension to $r<0$ but not for $r \rightarrow 0$. This plot refers to the Type I but for the Type II is almost equal.

\section{THE FULL-POLYMERIC SPINNING LBH}

In this section we apply the Newmann-Janis [? transformation to the loop black hole metric in its full polimeric form, (3). This metric is quite complicated than the semi-polymeric one, thus we restrict our analysis to more features independent from the complexification of the function $H(r)$. Moreover we restrict ourself to a particular complexification of the functions, even if suggested by the complexification of the Reissner-Nordström metric. The full polimeric line element can be rewritten as follows,

$$
\begin{aligned}
& d s^{2}=g_{t t} d t^{2}+g_{r r} d r^{2}+g_{\Omega} d \Omega^{(2)}, \\
& g_{t t}:=e^{2 \Phi(r)}=\left(1-\frac{r_{+}+r_{-}}{r}+\frac{r_{+} r_{-}}{r^{2}}\right)\left(1+\frac{r_{*}}{r}\right) \frac{r^{2}}{H(r)}, \\
& g_{r r}:=-e^{2 \lambda(r)}=-\frac{\left(1+\frac{r_{*}}{r}\right)^{2}}{\left(1-\frac{r_{+}+r_{-}}{r}+\frac{r_{+} r_{-}}{r^{2}}\right)} \frac{H(r)}{r^{2}}, \\
& g_{\Omega}:=-H(r)=-\left(r^{2}+\frac{a_{o}^{2}}{r^{2}}\right)
\end{aligned}
$$

where $r_{+}=2 m$ and $r_{-}=2 m P^{2}$ and $r_{*}=2 m P$ as already defined in the first section of the paper. The terms of the form $1 / r, 1 / r^{2}$ and $r^{2}$ are naturally complexified as the Reissner-Nordström terms, leaving us the choice, as for the semi polimeric case, to complexify the function $H(r)$,

$$
\begin{aligned}
& \frac{1}{r} \mapsto 2\left(\frac{1}{r^{\prime}}+\frac{1}{\bar{r}^{\prime}}\right), \\
& \frac{1}{r^{2}} \mapsto \frac{1}{r^{\prime} \bar{r}^{\prime}}, \\
& r^{2} \mapsto r^{\prime} \bar{r}^{\prime} .
\end{aligned}
$$


The line element in Kerr coordinates is

$$
\begin{aligned}
& d s^{2}=e^{2 \Phi} d u^{2}+2 e^{\lambda+\Phi} d u d r-2 a \sin ^{2} \theta e^{\lambda+\Phi} d r d \phi \\
& -\Sigma d \theta^{2}-\sin ^{2} \theta\left(\Sigma+a^{2} \sin ^{2} \theta\left(2 e^{\lambda+\Phi}-e^{2 \Phi}\right)\right) d \phi^{2} \\
& +2 a \sin ^{2} \theta\left(e^{\lambda+\Phi}-e^{2 \Phi}\right) d u d \phi
\end{aligned}
$$

where $\Sigma$ comes from the complexification of $H(r)$ and

$$
\begin{aligned}
& e^{2 \Phi(r, \theta)}=\frac{e^{\lambda(r, \theta)+\Phi(r, \theta)}\left(\rho^{2}(r, \theta)-\left(r_{+}+r_{-}\right) r+r_{-} r_{+}\right)}{\Sigma(r, \theta)} \\
& e^{\lambda(r, \theta)+\Phi(r, \theta)}=\left(1+\frac{r r_{*}}{\rho^{2}(r, \theta)}\right)^{2}
\end{aligned}
$$

and $\rho^{2}:=r^{2}+a^{2} \cos ^{2} \theta$ is the same function introduced in the semi-polymeric case.

We show now the regularity of the solution considering the Type I complexification of $H(r) \rightarrow \Sigma(r, \theta)$. We can rewrite the metric (51) in a conformal shape where the conformal factor is $\exp (\Phi+\lambda):=\exp (2 \sigma)$. The metric reads

$$
g_{\mu \nu}=e^{\Phi+\lambda} \bar{g}_{\mu \nu}:=e^{2 \sigma} \bar{g}_{\mu \nu} .
$$

where $\bar{g}_{\mu \nu}$ is regular $\forall r \geqslant 0$ (this is very simple to see for the Type I complexification because the components of the metric $\bar{g}_{\mu \nu}$ never diverge for $\theta=\pi / 2$ ) and presents the usual bounce of the two-sphere in $r=0$. Now we consider the Ricci scalar which can be written in the following way

$$
R=e^{-2 \sigma}(\bar{R}+6(\bar{\nabla} \sigma))
$$

where $\bar{R}, \bar{\nabla}$ are defined by $\bar{g}_{\mu \nu}$. When we replace the components of the metric in (53) for $\theta=\pi / 2$ we find the following leading term

$$
R \approx e^{-2 \sigma} 6(\bar{\nabla} \sigma) \approx \frac{6 a^{2}}{a_{o}^{2}}
$$

which shows the Ricci invariant does not diverges on the equatorial plane for $r=0$. The behavior of the Ricci scalar (53) is a strong argument in favor the regularity of the metric $g_{\mu \nu}$ for $\theta \approx \pi / 2$ and $r \geqslant 0$. Another argument pro regularity of the space-time comes from the radial geodesic analysis (for $r \approx 0$ ) in the Boyer-Lindquist coordinates we are going to introduce. We will return on this point having introduced such coordinates.

The components of the loop improved Kerr metric can be written in Boyer-Lindquist coordinates applying the transformation (25). The result is

$$
\begin{aligned}
& g_{t t}=\frac{\left(\Delta(r)+a^{2} \cos ^{2} \theta\right)\left(\rho^{2}+r r_{*}\right)^{2}}{\rho^{4} \Sigma} \\
& g_{r r}=-\frac{\Sigma\left(\rho^{2}+r r_{*}\right)^{2}}{\rho^{4}\left(\Delta(r)+a^{2} \cos ^{2} \theta\right)+a^{2} \sin ^{2} \theta\left(\rho^{2}+r r_{*}\right)^{2}} \\
& g_{t \phi}=\frac{a \sin ^{2} \theta\left(\rho^{2}+r r_{*}\right)^{2}\left[\Sigma-\left(\Delta(r)+a^{2} \cos ^{2} \theta\right)\right]}{\Sigma \rho^{4}} \\
& g_{\theta \theta}=-\Sigma, \\
& g_{\phi \phi}=-\sin ^{2} \theta[\Sigma \\
&\left.+a^{2} \sin ^{2} \theta \frac{\left(\rho^{2}+r r_{*}\right)^{2}\left(2 \Sigma-\left(\Delta(r)+a^{2} \cos ^{2} \theta\right)\right)}{\Sigma \rho^{4}}\right],
\end{aligned}
$$

where $\Sigma(r, \theta)$ is the complexification of $H(r)$ and we introduced the notation

$$
\Delta(r)=r^{2}-\left(r_{+}+r_{-}\right) r+r_{+} r_{-} .
$$

The ergosphere is quite similar to the classical one and is defined by the surface

$$
g_{t t}=0 \rightarrow \Delta(r)+a^{2} \cos ^{2} \theta=0
$$

or more explicitly

$$
\begin{aligned}
& r=\frac{r_{+}+r_{-} \pm \sqrt{\left(r_{+}-r_{-}\right)^{2}-4 a^{2} \cos ^{2} \theta}}{2} \\
& =m\left(1+P^{2}\right) \pm \sqrt{m^{2}\left(1-P^{2}\right)^{2}-a^{2} \cos ^{2} \theta} .
\end{aligned}
$$

The event horizon is defined by (30) and such relation for the full polymeric metric reads

$$
\rho^{4}\left(\Delta(r)+a^{2} \cos ^{2} \theta\right)+a^{2} \sin ^{2} \theta\left(\rho^{2}+r r_{*}\right)^{2}=0 .
$$

The definition of black hole horizon we are using here is the following: it is a surface within all lightlike paths and hence all paths in the forward light cones of particles within the horizon, are warped so as to fall farther into the hole. Equation (59) defines a null surface as it is easy to see.

Contour-plots of the six order equation (59) are given in Fig 1415. On the $x, y$ axes are $r$ and $\theta$ on the $z$ axes is the angular momentum $a$. The horizontal plane for constant $a$ shows explicitly that for small values of $a$ we have two quasi-spherical event horizons (Fig 15) one inside the other but for a sufficient large $a$ we have two horizons separated from each other (Fig, 14). For the second configuration the following picture follows: from an observer outside the hole the black hole splits along the symmetry axes in two distinct black holes both with ellipsoidal horizon. In the presence of two horizons, one inside the other, or zero horizons the Penrose diagrams (for $\theta=0$ ) are the same of Type I or Type II respectively depending on the complexification of $H(r)$. In the case of two topologically distinct event horizon the Penrose diagram representation is not well define. Indeed, in this case we 


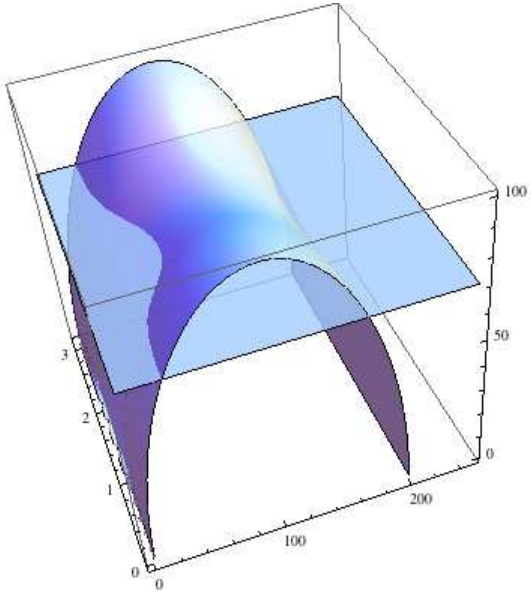

FIG. 14: Contour-plot for the geometric surface where equation (59) is satisfied. The intersecting plane is $a=$ const.. This plot refers to the case of two event horizon one inside the other for $m=100$ in Planck units and $P=0.1$.

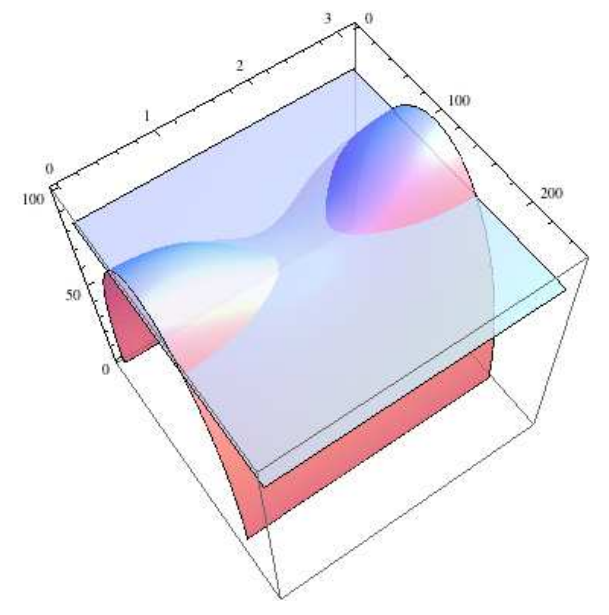

FIG. 15: Contour-plot for the geometric surface where equation (59) is satisfied. The intersecting plane is $a=$ const.. This plot shows the new phenomenon of two distinct horizons for $m=100$ in Planck units and $P=0.1$.

have two black hole each with a single event horizon but both inside the same ergosphere.

Another elegant way to verify the non existence of singularity in $r=0$ for $\theta=\pi / 2$ is to study the geodesic in the equatorial plane. We have a singularity problem only if the proper time to arrive in $r=0$ is finite. For the metric (55) on the plane $\theta=\pi / 2$ orbits are parametrized by the conserved energy per unit of mass, $E$, and the angular momentum per unit of mass along the symmetry axis the radial geodesic of a massive particle can be obtained from the norm of the 4-velocity and the conserved quantities associated with the symmetries of the

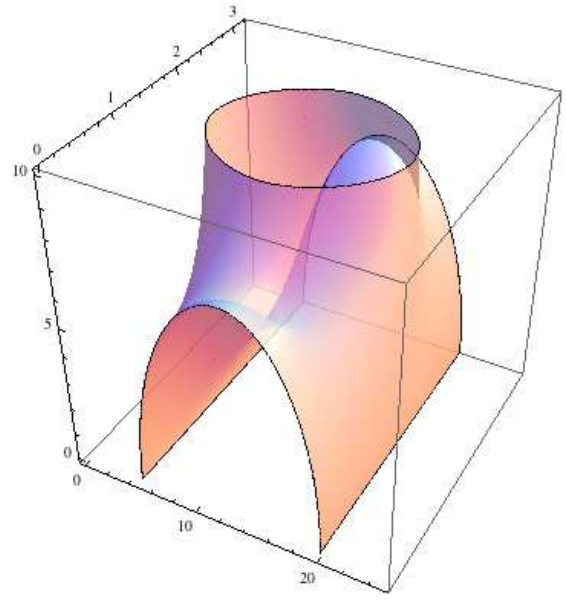

FIG. 16: Contour-plot for the geometric surface where the event horizon equation (59) is satisfied together with the ergosphere surface. The event horizon surface is always inside the ergosphere surface and the two surface meet at the poles $\theta=0, \pi$. In this plot $m=10$ in Planck units and $P=0.5$ but it is true for any value of the parameters.

metric

$$
\begin{aligned}
& E=t^{\mu} U^{\nu} g_{\mu \nu}, \quad \ell=-\phi^{\mu} U^{\nu} g_{\mu \nu} \\
& U^{\mu} U_{\mu}=1
\end{aligned}
$$

where we recall the Killing vectors $t^{\mu}=(1,0,0,0)$ and $\phi^{\mu}=(0,0,0,1)$. The second equation on (60) for general value of $E, \ell$ and small value of $r$ reads

$$
\frac{1}{2}\left(\frac{d r}{d \tau}\right)^{2} \approx-\frac{a^{2} r^{2}}{2 a_{o}^{2}}-\frac{\left(a E \ell+r_{-} r_{+}\right)}{2 a_{o}^{2} r_{*}^{2}} r^{4}
$$

since the leading term is negative for any value of $E$ and $\ell$ we conclude there is a barrier which prevent to arrive in $r=0$ both for positive and negative values or $r$. In the classical case a test particle can arrive in $r=0$ only from $r<0$ because $\dot{r}^{2} \propto-1 / r^{5}(\dot{r}$ is the derivative respect to the proper time $\tau$ ). Any test particle will move around the $\operatorname{ring}(r=0, \theta=\pi / 2)$ without ever reach it. Moreover, for $\theta=0$ any particle arrive in $r=0$ in finite time and then is natural an analytical extension to negative values of the coordinate $r$.

This is a strong argument in favor of the regularity of the space-time in $r=0$ but not exclude the possibility to have singularities for negative values of $r$. We can indeed to reach the region $r<0$ starting from $\theta \neq \pi / 2$. This result is independent from the Type I or Type II complexification because for $\theta=\pi / 2$ the two different complexification of $\Sigma$ coincides. On the other side for $\theta \neq \pi / 2$ there is no singularity because the presents of the angular momentum $a$ in $\Sigma$ and in $\rho^{2}$. 


\section{A. Violation of causality}

We showed in the previous section that any observer on the equatorial plane never reaches the origin $r=0$. This result is important in relation to the CTCs because those curves exist only for negative values of $r$ which in this metric are not part of the maximal extension for $\theta=\pi / 2$. We can conclude that there are no CTCs for an observer arrives in $r=0$ traveling on the plane $\theta=\pi / 2$ because the maximal extension does not involve $r<0$. What about the region $r \approx 0$ and $\theta \approx \pi / 2$ ? We consider the Killing vector along the $\phi$ direction. This vector has norm $\phi^{\mu} \phi_{\mu}=g_{\phi \phi}$. We compare such norm in the classical case with the new regular metric. Let $r / a=\delta$ (small) and consider $\theta=\pi / 2+\delta$. Then classically

$$
\phi_{\mu} \phi^{\mu}=g_{\phi \phi}=-\frac{a m}{\delta}-a^{2}+\mathcal{O}(\delta)
$$

which is positive for sufficiently small and negative $\delta$. For the loop black hole and complexification Type I and Type II respectively we find

$$
\begin{aligned}
& \phi_{\mu} \phi^{\mu}=-\frac{2 a_{0}^{2}+a^{2} r_{*}^{2}}{2 a^{2} \delta^{2}}-\frac{2 a r_{*}}{\delta}+\text { const. }+\mathcal{O}(\delta), \\
& \phi_{\mu} \phi^{\mu}=-\frac{a_{0}^{2}+a^{2} r_{*}^{2}}{2 a^{2} \delta^{2}}-\frac{2 a r_{*}}{\delta}+\text { const. }+\mathcal{O}(\delta)
\end{aligned}
$$

which are always negative for small values of $\delta$. We conclude there are no CTC in the region around $r \approx 0$ and $\theta \approx \pi / 2$ contrary to the classical Kerr space-time.

Also for the full-polymeric metric like for the semipolymeric one we have a region, for negative values of $r$, where the norm $\phi^{\mu} \phi_{\mu}$ changes sign. We have a good improvement respect to the classical metric but we still have CTC curves.

\section{B. Horizon transitions}

The metric we have introduced shown that there is, if we align the surface with $a$ as $z$ axis, a relation between the critical points of the surface $C(r, \theta ; a)=0$, defined by (59), and the transitions of the null surface. In the following we will refer to this surface as critical surface. In this section we will try to make this relation precise on a more general setting, relying only on the properties of the surface for a generic perturbation of the critical surface. In particular we assume there is a situation similar to the one of the previous section: a rotating black hole not in vacuum according to the Einstein equations but that in the limit of some parameters going to zero (and that we consider small in general), the metric we obtain is Kerr. The previous example catches general peculiarities enough to generalize it. The only assumption is that such corrections of the Kerr metric do not break the main symmetries, that is, the spacetime has a symmetry axis along which rotations keep the metric invariant.
The first thing to note is that the surface is embedded in three dimensions and can be Taylor expanded around the critical points:

$$
\begin{gathered}
C\left(r \approx r_{c}, \theta \approx \theta_{c} ; a\left(r \approx r_{c}, \theta \approx \theta_{c}\right)\right) \\
=C\left(r_{c}, \theta_{c} ; a\left(r_{c}, \theta_{c}\right)\right)+c_{1} r^{2}+c_{2} \theta^{2}+O\left(r^{3}, \theta^{3}\right),
\end{gathered}
$$

where $c_{1}$ and $c_{2}$, normalized to \pm 1 are called the Morse indices of the surface critical point. In general however we have to extend the notion of criticality also to points that cannot have an expansion of this form, like the points lying on $\theta=0$ or $\theta=\pi$, so to the notion of absolute minimum and absolute maximum. Of course, if local critical points, there are three distinct possibilities: maxima, minima and saddle points corresponding to $\left(c_{1}=-1, c_{2}=-1\right),\left(c_{1}=1, c_{2}=1\right)$ and $\left(c_{1}=1, c_{2}=-1\right)$ respectively. These indices are fundamental to study the topological properties of surfaces in Morse theory. However in our case the problem is slightly different due to the invariance of this surface respect with the $\phi$ variable, which corresponds to a global $\mathcal{M} \times \mathcal{S}$ topology, where $\mathcal{M}$ is the manifold given by the intersection of the $a=$ const. surface and the critical surface, as in Fig 16 and $\mathcal{S}$ is a circle. In the following we will refer to tori and spheres to surfaces having the topology of tori and spheres respectively. It is easy to understand that in general local maxima coincide with shrinking, saddle points with splitting, and local minima create spherical null surfaces, as the angular momentum, the parameter $a$, increases. Absolute minima and maxima occuring at $\theta_{0}=0$ and thus $\theta_{0}=\pi$ are slightly different as we explain now. In fact the critical properties of the null surface is the global one; we have to translate the criticality of $\mathcal{M}$ in the criticality of $\mathcal{M} \times \mathcal{S}$. The points $\theta=0$ and $\theta=\pi$ are different because they have to be identified, since they lie on the axis of symmetry; moreover due to the axial symmetry, critical points at an angle $0<\theta_{0}<\pi$ have an identical critical points at $\theta_{0}^{\prime}=\pi-\theta_{0}$. By the Weierstress theorem to two local maxima coincide at least a minimum that, in this case, coincide with a saddle point in between the two local maxima. In general local saddle points correspond to splits: when the surface $a=$ const hit a saddle point the null surface splits in two parts: at the angle where there is the saddle point the null surface bends and when the $a=$ const. surface hits the saddle point and the null surface separates. The way how this happens depends on the nature of the critical surface. We can apply the same analisys for local maxima. The surface at constant angular momentum cuts local maxima of the critical surface on cicles $^{1}$ if these are not at the extremal points, which correspond to a tori for the null surface, being $\mathcal{S} \times \mathcal{S}$. Thus local maxima in general correspond to shrinking of tori or spheres if the maxima are at the extremal at increasing angular momentum.

\footnotetext{
${ }^{1}$ With the topology of a circle.
} 
When the surface $a=$ const hits the critical point the tori shrinks to a point and the null surface disappears. The same happens for the maxima occurring at the extremal points. However a local minimum at the extremal points of the critical surface is tricky. In fact, when it occurs, the surfaces of the inner and outer horizons bend and touch on the axis. If, for example, the local minima at the extremal points are the first occurring the overall topology assumes a toroidal shape, transforming the inner and outer horizons in a torus. Local minima instead are the opposite of local maxima, when the surface $a=$ const hit them they create spherical null surfaces. This analysis complete the picture, in the approximation of little corrections to the Kerr metric, of transitions from the inner-outer horizon to null-surfaces free spacetime.

\section{TOWARDS SPINNING LBH WITH CHARGE}

In this section we consider a black with spin and electric charge. First we introduce the generalization of the Schwarzschild LBHs (semi-polymeric and full-polymeric) to the Reissner-Nordström LBHs then we apply again the Newmann-Janis complex transformation to obtain rotating and charged LBHs.

\section{A. Reissner-Nordström LBH}

It is easy to extend the spherically symmetric LBHs to the case of a black hole with charge. We consider first the semi-polymeric case and then the full-polymeric case.

\section{Semi-polymeric case}

We recall the semi-polymeric metric for the spherically symmetric case without charge

$$
\begin{aligned}
& d s^{2}=\frac{r^{2}-2 m r}{H(r)} d t^{2}-\frac{d r^{2}}{\frac{r^{2}-2 m r}{H(r)}}-H(r) d \Omega^{(2)}, \\
& H(r)=r^{2}+\frac{a_{o}^{2}}{r^{2}} .
\end{aligned}
$$

Is very simple to introduce the charge and to obtain a regular metric with the correct classical limit. This can be done by the replacement [12]

$$
2 m r \rightarrow 2 m r-e^{2}
$$

in (65), where $e$ is the electric charge. The metric is very close to the Reissner-Nordström but with a bounce of the $S^{2}$ sphere on a minimum area $a_{o}$ which solve the singularity problem. It is easy to show going through the analysis in [4] the regularity of the metric for any value, positive negative or zero, of the radial coordinate.

\section{Full-polymeric case}

The generalization of the full-polymeric LBH to a charged black hole is also very simple. We recall again the metric

$$
\begin{aligned}
& d s^{2}=g_{t t} d t^{2}+g_{r r} d r^{2}-H(r) d \Omega^{(2)}, \\
& g_{t t}=\frac{\left(r^{2}-\left(r_{+}+r_{-}\right) r+r_{+} r_{-}\right)}{H(r)}\left(1+\frac{r_{*}}{r}\right)^{2}, \\
& g_{r r}:=-\frac{H(r)}{\left(r^{2}-\left(r_{+}+r_{-}\right) r+r_{+} r_{-}\right)}\left(1+\frac{r_{*}}{r}\right)^{2}, \\
& H(r)=r^{2}+\frac{a_{o}^{2}}{r^{2}} .
\end{aligned}
$$

In this case we replace

$$
\left(r_{+}+r_{-}\right) r \rightarrow\left(r_{+}+r_{-}\right) r-e^{2},
$$

The horizons are now located in

$$
\tilde{r}_{ \pm}=\frac{\left(r_{+}+r_{-}\right) \pm \sqrt{\left(r_{+}+r_{-}\right)^{2}-4\left(e^{2}+r_{+} r_{-}\right)}}{2} .
$$

It is easy to show going through the analysis in [4] the regularity of the metric for any value of the radial coordinate. Radial geodesic's analysis shows that we can not reach the center $r=0$ in finite time like in case of $e=0$.

For $m(1-P)^{2} \geqslant e$ we can express the metric in the following way

$$
\begin{aligned}
& g_{t t}=\frac{\left(r^{2}-\left(\tilde{r}_{+}+\tilde{r}_{-}\right) r+\tilde{r}_{+} \tilde{r}_{-}\right)}{H(r)}\left(1+\frac{\tilde{r}_{*}}{r}\right)^{2}, \\
& g_{r r}:=-\frac{H(r)}{\left(r^{2}-\left(\tilde{r}_{+}+\tilde{r}_{-}\right) r+\tilde{r}_{+} \tilde{r}_{-}\right)}\left(1+\frac{\tilde{r}_{*}}{r}\right)^{2}, \\
& H(r)=r^{2}+\frac{a_{o}^{2}}{r^{2}}
\end{aligned}
$$

where we have introduced $\tilde{r}_{*}=\tilde{r}_{+} \tilde{r}_{-}$if we want to keep the duality property of the metric. It is easy to see that the Reissner-Nordström full-polymeric LBH has exactly the same shape of the Schwarzschild full-polymeric LBH with $r_{+}, r_{-}$and $r_{*}$ replaced by $\tilde{r}_{+}, \tilde{r}_{-}$and $\tilde{r}_{*}$ at least for $m(1-P)^{2} \geqslant e$; this makes very easy to derive the full-polymeric Kerr-Newmann space-time.

\section{B. Kerr-Newmann LBH}

In this section we apply the Newmann-Janis complexification to the Reissner-Nordström LBH in its semipolymeric and full-polymeric form. The following derivation is justified by the decoupling between polymerization of the space and electric field. This is easy to see in the semi-polymeric case but a conjecture in the fullpolymeric one. 


\section{Semi-polymeric case}

The complexification is straightforward and following section IVA the natural choose of $G$ and $H$ are

$$
\begin{aligned}
& G(r) \rightarrow \frac{\rho^{2}-2 m r+e^{2}}{H(r)}, \\
& H(r) \rightarrow \rho^{2}+\frac{a_{o}^{2}}{r^{2}} \text { for Type I, } \\
& H(r) \rightarrow \rho^{2}+\frac{a_{o}^{2}}{\rho^{2}} \text { for Type II, }
\end{aligned}
$$

where $\rho^{2}=r^{2}+a^{2} \cos ^{2} \theta$. The Kerr-Newmann LBH in Boyer-Lindquist coordinates reads

$$
\begin{aligned}
& d s^{2}=\frac{\Delta-a^{2} \sin ^{2} \theta}{\Sigma} d t^{2}-\frac{\Sigma}{\Delta} d r^{2} \\
& +2 a \sin ^{2} \theta\left(1-\frac{\Delta-a^{2} \sin ^{2} \theta}{\Sigma}\right) d t d \phi-\Sigma d \theta^{2} \\
& -\sin ^{2} \theta\left[\Sigma+a^{2} \sin ^{2} \theta\left(2-\frac{\Delta-a^{2} \sin ^{2} \theta}{\Sigma}\right)\right] d \phi^{2}
\end{aligned}
$$

where now $\Delta(r)$ is

$$
\Delta(r)=r^{2}-2 m r+e^{2}+a^{2} .
$$

The rest of the analysis follows exactly section IVA and IVB but with the new $\Delta(r)$ function defined in (73). Particularly for the Type I complexification, $\Omega_{H}, \kappa_{ \pm}$and the event horizon area have the same shape as those calculated in sectionIVA but with $r_{ \pm}$replaced with the roots of the new equation $\Delta(r)=0$ defined in (73).

\section{Full-polymeric case}

This section is very short since it is identical to $\mathrm{V}$ if we replace everywhere $r_{+}, r_{-}$and $r_{*}$ with $\tilde{r}_{+}, \tilde{r}_{-}$and $\tilde{r}_{*}$ when $m(1-P)^{2} \geqslant e$. If $m(1-P)^{2}<e$ we still define $\tilde{r}_{*}^{2}=\tilde{r}_{+} \tilde{r}_{-}=e^{2}+r_{+} r_{-}$but we apply the NewmannJanis transformation directly to (67) with the replacement (68).

\section{CONCLUSIONS}

In this paper we used the Newman-Janis algorithm to construct regular spinning black hole from the Schwarzschild loop black hole. We used constraints coming from the classical limits and arguments from the Newman-Janis algorithm applied in the past to the Schwarzshild metric and The Reissner-Nordström metric. We found Kerr-like geometries without ring singularity. These results, while not definitive, hints in the direction that the polimeric quantization inspired by loop quantum gravity could solve the singularity problem also for the Kerr spacetime. We started considering two different spherically symmetric space-time obtained in [4] that we called semi-polymeric and full-polymeric. The first metric can be obtained from the second one in an appropriate limit. We studied the semi-polymeric one for reasons of pure simplicity since such metric has all good property of regularity. In the paper we introduced also the notation Type I and Type II to indicate the two complexifications we used. For the semi-polymeric spinning loop black hole we showed explicitly that the Ricci scalar and the Kretschmann invariant are regular in $r=0$ and $\theta=\pi / 2$ (for the semi-polymeric Type I loop black hole the reader can fine the explicit formula for the Ricci scalar and the Kretschmann invariant). The structure of the event horizon and of the ergosphere is the same of the classical Kerr metric and the causal space-time structure is given in the text for each case in terms of Penrose diagrams. The full-polymeric spinning loop black hole has a more reach structure. The ergosphere surfaces are very similar to the classical ones but the horizon surfaces, while are very similar to the classical ones for small values of the angular momentum, change topology for large value of the angular momentum respect to the mass. The singularity here is also cured but in a more elegant way. Any observer in the equatorial plane $(\theta=\pi / 2)$ never can reach the point $r=0$ starting from positive or negative values of the radial coordinate. Of course the Ricci scalar and the Kretschmann invariant are regular.

For the first time we introduce the Reissner-Nordström LBH metric and extended the Newmann-Janis transformation to this to obtain the Kerr-Newmann LBH with spin and electric charge. The properties of the spinning LBHs are shared by the spinning and charged LBHs. For all semi-polymeric cases studied there are no naked singularities for any value of the angular momentum.

We studied the presence of CTCs (closed timelike curves) in the region near $r \approx 0$ and $\theta \approx \pi / 2$ and we have shown CTCs disappear in all the new metrics. In particular for the full polymeric since each observer never can arrive in $r=0$ there is no physical reason to extend the space-time to negative values of $r$ where classically the CTCs are located. This result does not exclude the existence of other CTC's regions for negative values of $r$ where $g_{\phi \phi}$ changes sign.

In this paper we did not solve the equations of motion coming from a fundamental theory but we simply introduced the angular momentum in spherically symmetric solutions by the Newmann-Janis transformation. However we can always see spherically symmetric LBHs to be solutions of the Einstein theory with an effective energy tensor: $G_{\mu \nu}=8 \pi T_{\mu \nu}^{\mathrm{QG}}$; where $T_{\mu \nu}^{\mathrm{QG}}$ summarizes the loop corrections. The Spinning LBHs obtained in this paper are actually solutions of the Einstein equations with a stress energy tensor obtained from the spherically symmetric one applying the Newmann-Janis transformation properly. The effective stress energy tensor is a function of two or three parameters depending on the semi- 
polymeric or full-polymeric nature of the LBH:

$G_{\mu \nu}=8 \pi \begin{cases}T_{\mu \nu}^{\mathrm{QG}}(a, P), & \text { SEMI - POLYMERIC }, \\ T_{\mu \nu}^{\mathrm{QG}}\left(a, P, a_{o}\right), & \text { FULL - POLYMERIC } .\end{cases}$
We conclude the paper summarizing the metrics obtained.

\section{SEMI-POLYMERIC :}

$$
\begin{gathered}
d s^{2}=\frac{\Delta-a^{2} \sin ^{2} \theta}{\Sigma} d t^{2}-\frac{\Sigma}{\Delta} d r^{2}-\Sigma d \theta^{2} \\
+2 a \sin ^{2} \theta\left(1-\frac{\Delta-a^{2} \sin ^{2} \theta}{\Sigma}\right) d t d \phi-\sin ^{2} \theta\left[\Sigma+a^{2} \sin ^{2} \theta\left(2-\frac{\Delta-a^{2} \sin ^{2} \theta}{\Sigma}\right)\right] d \phi^{2}, \\
\Delta(r)=r^{2}-2 m r+a^{2} .
\end{gathered}
$$

\section{FULL-POLYMERIC :}

$$
\begin{aligned}
& d s^{2}=\frac{\left(\Delta-a^{2} \sin ^{2} \theta\right)\left(\rho^{2}+r r_{*}\right)^{2}}{\rho^{4} \Sigma} d t^{2}-\frac{\Sigma\left(\rho^{2}+r r_{*}\right)^{2}}{\rho^{4}\left(\Delta-a^{2} \sin ^{2} \theta\right)+a^{2} \sin ^{2} \theta\left(\rho^{2}+r r_{*}\right)^{2}} d r^{2}-\Sigma d \theta^{2} \\
& +2 \frac{a \sin ^{2} \theta\left(\rho^{2}+r r_{*}\right)^{2}\left[\Sigma-\left(\Delta-a^{2} \sin ^{2} \theta\right)\right]}{\Sigma \rho^{4}} d t d \phi-\sin ^{2} \theta\left[\Sigma+a^{2} \sin ^{2} \theta \frac{\left(\rho^{2}+r r_{*}\right)^{2}\left(2 \Sigma-\left(\Delta-a^{2} \sin ^{2} \theta\right)\right)}{\Sigma \rho^{4}}\right] d \phi^{2}, \\
& \Delta=r^{2}-\left(r_{+}+r_{-}\right) r+r_{+} r_{-}+a^{2},
\end{aligned}
$$

Type I : $\quad \Sigma=r^{2}+a^{2} \cos ^{2} \theta+\frac{a_{o}^{2}}{r^{2}}$,

Type II : $\quad \Sigma=r^{2}+a^{2} \cos ^{2} \theta+\frac{a_{o}^{2}}{r^{2}+a^{2} \cos ^{2} \theta}$.

The function $\Sigma$ is the same for both the metrics.

\section{Acknowledgments}

The authors are indebted with S.P. Drake for clarifications on the Newman-Janis algorithm and Roberto Balbinot for the useful comments on the black hole thermodynamics. Research at Perimeter Institute is supported by the Government of Canada through Industry Canada and by the Province of Ontario through the Ministry of Research \& Innovation.

\section{APPENDICES}

\section{A. Kretschmann invariant for the Type I semi-polymeric LBH}

The Kretschmann invariant for the Type I complexified metric reads

$$
\begin{aligned}
& K(r, \theta)=\frac{16}{\left(2 r^{4}+a^{2} r^{2}+a^{2} \cos (2 \theta) r^{2}+2 a_{o}^{2}\right)^{6}} \times \\
& \left(192 m^{2} r^{18}-1440 a^{2} m^{2} r^{16}+1536 a_{o}^{2} m r^{15}+1080 a^{4}\right.
\end{aligned}
$$

$$
\begin{aligned}
& m^{2} r^{14}-3072 a_{o}^{2} m^{2} r^{14}-2880 a^{2} a_{o}^{2} m r^{13}+4032 a_{o}^{4} r^{12} \\
& -60 a^{6} m^{2} r^{12}+7296 a^{2} a_{o}^{2} m^{2} r^{12}-6 a^{6} m^{2} \cos (6 \theta) r^{12} \\
& -17408 a_{o}^{4} m r^{11}-1536 a^{4} a_{o}^{2} m r^{11}+5328 a^{2} a_{o}^{4} r^{10} \\
& +18816 a_{o}^{4} m^{2} r^{10}-288 a^{4} a_{o}^{2} m^{2} r^{10}-10464 a^{2} a_{o}^{4} m r^{9} \\
& -312 a^{6} a_{o}^{2} m r^{9}+12 a^{6} a_{o}^{2} m \cos (6 \theta) r^{9}-2176 a_{o}^{6} r^{8} \\
& +3192 a^{4} a_{o}^{4} r^{8}-160 a^{2} a_{o}^{4} m^{2} r^{8}+7168 a_{o}^{6} m r^{7}-3360 \\
& a^{4} a_{o}^{4} m r^{7}-672 a^{2} a_{o}^{6} r^{6}+1002 a^{6} a_{o}^{4} r^{6}-4608 a_{o}^{6} m^{2} r^{6} \\
& +336 a^{4} a_{o}^{4} m^{2} r^{6}+3 a^{6} a_{o}^{4} \cos (6 \theta) r^{6}-448 a^{2} a_{o}^{6} m r^{5} \\
& -396 a^{6} a_{o}^{4} m r^{5}-18 a^{6} a_{o}^{4} m \cos (6 \theta) r^{5}+704 a_{o}^{8} r^{4}+144 \\
& a^{4} a_{o}^{6} r^{4}+144 a^{8} a_{o}^{4} r^{4}+64 a^{2} a_{o}^{6} m^{2} r^{4}+9 a^{8} a_{o}^{4} \cos (6 \theta) r^{4} \\
& -1536 a_{o}^{8} m r^{3}+88 a^{4} a_{o}^{6} m r^{3}-176 a^{2} a_{o}^{8} r^{2}+48 a^{6} a_{o}^{6} r^{2} \\
& +960 a_{o}^{8} m^{2} r^{2}-32 a^{2} a_{o}^{8} m r+34 a^{4} a_{o}^{8}+a^{2}\left(207 a_{o}^{4} r^{4} a^{6}\right. \\
& +3\left(-30 m^{2} r^{12}-132 a_{o}^{2} m r^{9}+3 a_{o}^{4}(133 r-62 m) r^{5}\right. \\
& \left.+16 a_{o}^{6} r^{2}\right) a^{4}+8\left(180 m^{2} r^{14}-48 a_{o}^{2} m(m+4 r) r^{10}+2 a_{o}^{4}\right. \\
& \left.\left(28 m^{2}-216 r m+123 r^{2}\right) r^{6}+4 a_{o}^{6}(m-3 r) r^{3}+5 a_{o}^{8}\right) a^{2} \\
& -16 r\left(90 m^{2} r^{15}+12 a_{o}^{2} m(21 r-38 m) r^{11}+a_{o}^{4}\left(10 m^{2}\right.\right. \\
& \left.+26 r m-27 r^{2}\right) r^{7}-2 a_{o}^{6}\left(2 m^{2}+2 r m+r^{2}\right) r^{3}+a_{o}^{8} \\
& (30 m-19 r))) \cos (2 \theta)+2 a^{4}\left(18 m^{2}\left(10 r^{2}-a^{2}\right) r^{12}\right. \\
& -12 a_{o}^{2} m\left(3 a^{2}+4 m r\right) r^{9}+a_{o}^{4}\left(36 a^{4}+\left(99 r^{2}-90 m r\right) a^{2}\right. \\
& \left.+4 r^{2}\left(14 m^{2}-12 r m+9 r^{2}\right)\right) r^{4}-4 a_{o}^{6}(7 m-6 r) r^{3} \\
& \left.\left.+11 a_{o}^{8}\right) \cos (4 \theta)\right) .
\end{aligned}
$$


This quantity is regular and finite everywhere and in particular

$$
\lim _{r \rightarrow 0}\left(\lim _{\theta \rightarrow \pi / 2} K(r, \theta)\right)=\lim _{\theta \rightarrow \pi / 2}\left(\lim _{r \rightarrow 0} K(r, \theta)\right)=\frac{4 a^{4}}{a_{o}^{4}} .
$$

\section{B. Tortoise coordinates for the full polymeric metric}

The case of two distinct horizons with Type I complexification :

$$
\begin{aligned}
& r^{*}=r-\frac{a_{o}^{2}}{r r_{2} r_{1}}-\frac{\left(a^{2} r_{2}^{2}+a_{o}^{2}+r_{2}^{4}\right)}{r_{2}^{2}\left(r_{1}-r_{2}\right)} \log \left|r-r_{2}\right| \\
& +\frac{\left(a^{2} r_{1}^{2}+a_{o}^{2}+r_{1}^{4}\right)}{r_{1}^{2}\left(r_{1}-r_{2}\right)} \log \left|r-r_{1}\right|+\frac{\left(a_{o}^{2} r_{2}+a_{o}^{2} r_{1}\right)}{r_{2}^{2} r_{1}^{2}} \log |r|,
\end{aligned}
$$

where $r_{1,2}$ are the bigger and the smaller horizons for $\theta=0$ and then coincide with the roots in (58).
The case of no horizons at all and Type I complexification:

$$
\begin{aligned}
r^{*}=r & -\frac{a_{o}^{2}}{r\left(a^{2}+r_{-} r_{+}\right)}+\frac{a_{o}^{2} \log (r)\left(r_{-}+r_{+}\right)}{\left(a^{2}+r_{-} r_{+}\right)^{2}} \\
& +\frac{\left(r_{-}+r_{+}\right)\left(a^{4}+2 a^{2} r_{-} r_{+}-a_{o}^{2}+r_{-}^{2} r_{+}^{2}\right)}{2\left(a^{2}+r_{-} r_{+}\right)^{2}} \times \\
& \log \left(a^{2}+\left(r-r_{-}\right)\left(r-r_{+}\right)\right) \\
& +\frac{\arctan \left[\frac{2 r-r_{-}-r_{+}}{\sqrt{4 a^{2}-\left(r_{-}-r_{+}\right)^{2}}}\right]}{\sqrt{4 a^{2}-\left(r_{-}-r_{+}\right)^{2}}\left(a^{2}+r_{-} r_{+}\right)^{2}} \times \\
& {\left[a^{4}\left(r_{-}^{2}+r_{+}^{2}\right)+a^{2}\left(2 r_{-} r_{+}\left(r_{-}^{2}+r_{+}^{2}\right)-2 a_{o}^{2}\right)\right.} \\
& \left.+\left(r_{-}^{2}+r_{+}^{2}\right)\left(a_{o}^{2}+r_{-}^{2} r_{+}^{2}\right)\right] .
\end{aligned}
$$

[1] C. Rovelli, Quantum Gravity, (Cambridge University Press, Cambridge, 2004); A. Ashtekar, Background independent quantum gravity: A Status report, Class. Quant. Grav. 21, R53 (2004), gr-qc/0404018; T. Thiemann, Introduction to Modern Canonical Quantum General Relativity, [gr -qc/0110034]; A Perez, Introduction to loop quantum gravity and spin foams, gr-qc/0409061

[2] L Modesto, C Rovelli, Particle scattering in loop quantum gravity, Phys. Rev. Lett. 95, 191301 (2005) gr-qc/0502036; E Bianchi, L Modesto, C Rovelli, S Speziale, Graviton propagator in loop quantum gravity, Class. Quant. Grav. 23, 6989-7028 (2006) gr-qc/0604044; E Bianchi, E Magliaro, C Perini, Spinfoams in the holomorphic representation, arXiv:1004.4550 [gr-qc]]; E Bianchi, C Rovelli, F Vidotto, Towards Spinfoam Cosmology, arXiv:1003.3483 [gr-qc]]; E Bianchi, E Magliaro, C Perini, Coherent spinnetworks, arXiv:0912.4054 [gr-qc]]; F Conrady, L Freidel, On the semiclassical limit of $4 \mathrm{~d}$ spin foam models, Phys. Rev. D78, 104023 (2008) arXiv:0809.2280 [gr-qc]]; F Conrady, L Freidel, Path integral representation of spin foam models of 4d gravity Class. Quant. Grav. 25, 245010 (2008) arXiv:0806.4640 [gr-qc]]

[3] M Bojowald, Loop quantum cosmology, Living Rev. Rel. 8, 11, 2005 gr-qc/0601085; A. Ashtekar, M. Bojowald and J. Lewandowski, Mathematica structure of loop quantum cosmology, Adv. Theor. Math. Phys. 7 (2003) 233-268 gr-qc/0304074; M Bojowald, Absence of singularity in loop quantum cosmology, Phys. Rev. Lett. 86, 5227-5230, 2001 gr-qc/0102069

[4] L. Modesto, Space-Time Structure of Loop Quantum Black Hole, Int. J. Theor. Phys 2010 arXiv:0811.2196 [gr-qc]]

[5] L Modesto, I Premont-Schwarz Self-dual Black Holes in LQG: Theory and Phenomenology, Phys. Rev. D 80, 064041, 2009 arXiv:0905.3170 [hep-th]]; S Hossenfelder, L Modesto, I Premont-Schwarz, A Model for non-singular black hole collapse and evaporation. Phys.
Rev. D81, 044036, 2010 arXiv:0912.1823 [gr-qc]]; L Modesto, Black hole interior from loop quantum gravity. Adv. High Energy Phys. 2008, 459290, 2008 gr-qc/0611043; L Modesto, Loop quantum black hole, Class. Quant. Grav. 23, 5587-5602, 2006 gr-qc/0509078; A Ashtekar, $M$ Bojowald, Quantum geometry and Schwarzschild singularity, Class. Quant. Grav. 23 (2006) 391-411, gr-qc/0509075; L Modesto, Loop quantum black hole, Class. Quant. Grav. 23, 5587-5602 (2006), gr-qc/0509078; L Modesto, Disappearance of black hole singularity in quantum gravity, Phys. Rev. D 70, 124009, 2004 gr-qc/0407097; C G. Bohmer, K. Vandersloot, Loop quantum dynamics of the Schwarzschild Interior, arXiv:0709.2129 D W Chiou, Phenomenological Loop Quantum Geometry of the Schwarzschild Black Hole, Phys. Rev. D 78, 064040, 2008 arXiv:0807.0665; J Ziprick, G Kunstatter Quantum Corrected Spherical Collapse: A Phenomenological Framework, arXiv:1004.0525 [gr-qc]]; G Kunstatter, J Louko, A Peltola, Polymer quantization of the Einstein-Rosen wormhole throat, Phys. Rev. D81, 024034, 2010 arXiv:0910.3625 [gr-qc]]; J Ziprick, G Kunstatter, Dynamical Singularity Resolution in Spherically Symmetric Black Hole Formation, Phys. Rev. D80, 024032, 2009 arXiv:0902.3224 [gr-qc]]; A Peltola, G Kunstatter, Effective Polymer Dynamics of D-Dimensional Black Hole Interiors, Phys. Rev. D80, 044031, 2009 arXiv:0902.1746 [gr-qc]]; R Casadio, $\mathrm{P}$ Nicolini, The decay-time of non-commutative microblack holes, JHEP 0811, 072 (2008) arXiv:0809.2471 [hep-th]]

[6] R Gambini, J Pullin, Black holes in loop quantum gravity: The Complete space-time, Phys. Rev. Lett.101, 161301, 2008 arXiv:0805.1187]; M Campiglia, R Gambini, J Pullin, Loop quantization of spherically symmetric midi-superspaces : the interior problem, AIP Conf. Proc. 977, 52-63, 2008 arXiv:0712.0817]; M Campiglia, R Gambini, J Pullin, Loop quantization of spherically symmetric midi-superspaces Class. Quant. Grav. 24, 3649- 
3672, 2007 gr-qc/0703135; V Husain, D R Terno, Dynamics and entanglement in spherically symmetric quantum gravity, Phys. Rev. D 81, 044039, 2010 [e-Print: arXiv:0903.1471 [gr-qc]]

[7] A Smailagic, E Spallucci "Kerrr" black hole: the Lord of the String, arXiv:1003.3918; L Modesto, P Nicolini, Charged rotating noncommutative black holes, arXiv:1005.5605[gr-qc]]

[8] P Nicolini, A Smailagic and E Spallucci, The fate of radiating black holes in noncommutative geometry, arXiv:hep-th/0507226; P Nicolini, A model of radiating black hole in noncommutative geometry, J. Phys. A 38, L631 (2005) arXiv:hep-th/0507266; P Nicolini, A Smailagic and E Spallucci, Noncommutative geometry inspired Schwarzschild black hole, Phys. Lett. B 632, 547 (2006) arXiv:gr-qc/0510112; S. Ansoldi, P. Nicolini, A. Smailagic and E. Spallucci, Noncommutative geometry inspired charged black holes, Phys. Lett. B 645, 261 (2007) arXiv:gr-qc/0612035; E Spallucci, A Smailagic and $\mathrm{P}$ Nicolini, Non-commutative geometry inspired higher-dimensional charged black holes, Phys. Lett. B 670, 449 (2009) arXiv:0801.3519 [hep-th]]; P Nicolini and E Spallucci, Noncommutative geometry inspired wormholes and dirty black holes, Class. Quant. Grav. 27, 015010 (2010) arXiv:0902.4654 [gr-qc]]; P Nicolini, M Rinaldi, A minimal length versus the Unruh effect, arXiv:0910.2860 [hep-th]; M Bleicher and P Nicolini, "Large Extra Dimensions and Small Black Holes at the LHC, arXiv:1001.2211 [hep-ph]; D Batic, P Nicolini, Fuzziness at the horizon, arXiv:1001.1158 [gr-qc]]; Y S Myung, Y W Kim, Young-Jai Park, Thermodynamics of regular black hole, arXiv:0708.3145; Y. S. Myung, Y. W. Kim, Y. J. Park, Quantum Cooling Evaporation Process in Regular Black Holes, Phys. Lett. B (2007) 656:221-225, gr-qc/0702145] P. Nicolini, Noncommutative Black Holes, The Final Appeal To Quantum Gravity: A Review, arXiv:0807.1939; R. Banerjee, B. R. Majhi, S. Samanta, Noncommutative Black Hole Thermodynamics, Phys. Rev. D77 (2008) 124035, arXiv:0801.3583; R. Banerjee, B. R. Majhi, S. K. Modak, Area Law in Noncommutative Schwarzschild Black Hole, arXiv:0802.2176] V Husain, R B Mann, Thermodynamics and phases in quantum gravity, Class. Quant. Grav. 26, 075010 (2009) arXiv:0812.0399 [gr-qc]]

[9] E T Newman, A. Janis, J. Math. Phys. 6, 915 (1965); S. P. Drake, P. Szekeres, Gen.Rel.Grav. 32 (2000) 445458, [arXiv:9807001v1[gr-qc]]; L Herrera, J Jimenez, The complexification of a non rotating sphere: An extension of the Newman-Janis algorithm, Journal of Mathematical Physics 23, 2339-2345 (1982)

[10] M Walker, Block diagrams and extension of timelike twosurfaces, J. Math. Phys. 11, 2280 (1970)

[11] B O'Neil, The geometry of Kerr black holes, A K Peters Wellesley, Massachusetts, 1995

[12] H Andreka, I Nemeti, C Wuthrich, A Twist in the geometry of rotating black holes: Seeking the cause of acausality, Gen. Rel. Grav. 40, 1809-1823 (2008) arXiv:0708.2324 [gr-qc]] 